\title{
The finite volume element method for the two-dimensional space-fractional convection-diffusion equation
}

Yanan $\mathrm{Bi}^{1}$ and Ziwen Jiang ${ }^{1 *}$

Correspondence:

ziwenjiang@163.com

'School of Mathematics and

Statistics, Shandong Normal

University, Jinan, Shandong, China

\begin{abstract}
We develop a fully discrete finite volume element scheme of the two-dimensional space-fractional convection-diffusion equation using the finite volume element method to discretize the space-fractional derivative and Crank-Nicholson scheme for time discretization. We also analyze and prove the stability and convergence of the given scheme. Finally, we validate our theoretical analysis by data from three examples.
\end{abstract}

Keywords: Fractional derivative; Finite volume element method; Stability; Convergence; Convection-diffusion equation

\section{Introduction}

Fractional differential equations are generalizations of the integer-order differential equations; they contain noninteger-order derivatives and can effectively describe the memory and genetic properties of a variety of substances. Such equations play an increasingly important role in mathematical physics, mechanical applications, biological engineering, electronic science and technology, control theory, financial mathematics, and other fields [1-5].

Abnormal diffusion in physics originally developed from the random walk model [6-8]. Fractional convective diffusion equations are a powerful tool to simulate various abnormal diffusion phenomena. We consider the following one-dimensional random walk model. First, we denote by $n$ the random walk step size and assume that the waiting time obeys an exponential distribution with mean $\frac{\tau}{n}$ and the probability density function $\lambda(x)$ of the jump step obeys a stable distribution. We define the probability density function $W(x, t)$ of the particle at time $t$ at the position $x$. Then

$$
W(x, t)=\int_{0}^{t} \eta\left(x, t^{\prime}\right) \Psi\left(t-t^{\prime}\right) d t^{\prime}
$$

where $\Psi(t)=1-\int_{0}^{t} w\left(t^{\prime}\right) d t$ is the probability that the particle does not jump within the time interval $(0, t), \eta\left(x, t^{\prime}\right)$ is the probability that the particle is at the position of $x$ at time

(c) The Author(s) 2021. This article is licensed under a Creative Commons Attribution 4.0 International License, which permits use, sharing, adaptation, distribution and reproduction in any medium or format, as long as you give appropriate credit to the original author(s) and the source, provide a link to the Creative Commons licence, and indicate if changes were made. The images or other third party material in this article are included in the article's Creative Commons licence, unless indicated otherwise in a credit line to the material. If material is not included in the article's Creative Commons licence and your intended use is not permitted by statutory regulation or exceeds the permitted use, you will need to obtain permission directly from the copyright holder. To view a copy of this licence, visit http://creativecommons.org/licenses/by/4.0/. 
$t^{\prime}$ multiplied by the probability that the particle jumps $x-x^{\prime}$ step in time $t-t^{\prime}$, namely, the Markov supplement process in the random process $\eta(x, t)=\int_{-\infty}^{\infty} \int_{0}^{\infty} \eta\left(x^{\prime}, t^{\prime}\right) \psi\left(x-x^{\prime}, t-\right.$ $\left.t^{\prime}\right) d t^{\prime} d x^{\prime}+\delta(x) \delta(t)$, where $\delta(x) \delta(t)$ is the initial condition, and $\psi(x, t)=w(t) \lambda(x)$. Then taking the Laplace transform of $\Psi(t)$ and the Fourier and Laplace transforms of $x$ and $t$ in $W(x, t)$ and $\eta(x, t)$, respectively, we get

$$
\widetilde{\Psi}(s)=\frac{1-w(s)}{s}, \quad \widetilde{\widetilde{\eta}}(\kappa, s)=\frac{1}{1-\widetilde{\psi}(\kappa, s)}, \quad \widetilde{\widetilde{W}}(\kappa, s)=\frac{1-\widetilde{W}(s)}{s} \frac{1}{1-\widetilde{W}(s) \widehat{\lambda}(\kappa)}
$$

On the basis of the above, we discuss the random movement of particles in a fluid with mean velocity $V$ located in the porous medium. We assume that the particles are stuck in place while waiting for the next jump. Since the fluid is located in the porous medium, the particles may be confined to the pores, and so our hypothesis is reasonable. We denote by $n$ the number of random walk steps, by $\alpha$ the ratio index, by $\frac{\sigma^{\alpha}}{n}$ the diffusion coefficient, and by $\vartheta$ the tilt angle of the transition probability density function. Then we introduce the similar variable $\xi=x-V t$ to obtain the corresponding probability density function $\phi(x, t)=\psi(x-V t, t)$. In this case, the special function $\widehat{\lambda}(\kappa)=\exp ^{-|\kappa|^{\alpha}} \exp ^{i \operatorname{sign}(\kappa) \vartheta \pi / 2}=$ $1-\frac{|\kappa|^{\alpha} \exp ^{i \operatorname{sign}(\kappa) \vartheta \pi / 2}}{n}+O\left(\frac{1}{n}\right)$. After transformation and derivation, $\widetilde{W}(\kappa, s)$ can be rewritten as

$$
\widetilde{W}_{n}(\kappa, s)=\frac{1}{s+i V \kappa+K^{\alpha}\left(c_{+}(-i \kappa)^{\alpha}+c_{-}(i \kappa)^{\alpha}\right)+O(1)},
$$

where $K^{\alpha}=\frac{\sigma^{\alpha}}{\tau}, c_{+}=c_{+}(\alpha, \vartheta)=\frac{\sin ((\alpha-\vartheta) \pi / 2)}{\sin (\alpha \pi)}$, and $c_{-}=c_{-}(\alpha, \vartheta)=\frac{\sin ((\alpha+\vartheta) \pi / 2)}{\sin (\alpha \pi)}$. As $n \rightarrow \infty$, we get

$$
{ }_{s} \widetilde{W}_{n}(\kappa, s)=-K^{\alpha}\left(c_{+}(-i \kappa)^{\alpha}+c_{-}(i \kappa)^{\alpha}\right) \widetilde{W}_{n}(\kappa, s)-i V \kappa \widetilde{W}_{n}(\kappa, s) .
$$

Finally, we can obtain the following one-dimensional space fractional convectiondiffusion equation by the inverse Lapalce and Fourier transforms of the above equation:

$$
\frac{\partial W}{\partial t}=-K^{\alpha}\left(c_{+}(\alpha, \vartheta) \frac{d^{\alpha} W}{d x^{\alpha}}+c_{-}(\alpha, \vartheta) \frac{d^{\alpha} W}{d(-x)^{\alpha}}\right)-V \frac{\partial W}{\partial x}+\delta(x) \delta(t)
$$

When $\vartheta=0$ and $\alpha=1+\alpha_{1}\left(0<\alpha_{1}<1\right)$, equation (1) can be written in the form

$$
\frac{\partial W}{\partial t}=K^{1+\alpha_{1}} \frac{d^{1+\alpha_{1}} W}{d|x|^{1+\alpha_{1}}}-V \frac{\partial W}{\partial x}+\delta(x) \delta(t) .
$$

In this paper, we study the two-dimensional form of equation (2) with source terms. The finite volume element (FVE) method [9-13], also known as the generalized difference method, is important for solving differential equations. In recent years, more and more researchers used the finite volume element method to discretize fractional differential equations. In 2014, Liu et al. [14] used a new FVE method to give a fully discrete scheme for a class of one-dimensional space fractional diffusion equations with variable coefficients and nonlinear source terms. In 2015, Feng et al. [15] designed an implicit fractional finite vol- 
ume element scheme to solve the space fractional diffusion equation and proved the stability and convergence of the scheme. In 2017, Karaa et al. [16] constructed a fully discrete FVE scheme for the two-dimensional Riemann-Liouville fractional diffusion equation by using the piecewise linear discontinuous Galerkin method and FVE method and carried out error analysis and numerical calculation experiments. In 2019, Fu et al. [17, 18] used the CN-FVE method to give fully discrete finite volume element schemes for RiemannLiouville space fractional diffusion equations in one and two dimensions and adopted a new fast algorithm in numerical experiments. In 2020, Zhao et al. [19] proposed a fully discrete CN-FVE scheme to solve the Caputo time fractional Sobolev equation and verified the feasibility of this method. In the same year, Zhao et al. [20] used the WSGD formula to approximate the Riemann-Liouville fractional derivative and interpolation operator, established the second-order fully discrete FVE scheme for the nonlinear time fractional movable/immovable transport equation, and carried out stability analysis and error estimation of the scheme. In addition to the FVE method, researchers also used many other numerical methods to study fractional differential equations, such as the finite difference method [21-26], finite element method [27-31], spectral method [32-34], and so on [3540].

The main work we have done in this paper is obtaining a fully discrete finite volume element scheme of the two-dimensional Riesz space fractional convection-diffusion equation (3) by using the CN-FVE method. Also, we prove the uniqueness, stability, and convergence of the scheme in the $L^{2}$-norm. To save time, we consider the characteristics of Toplitz matrices, and use the FAST-BICGSTAB algorithm to solve the numerical examples, so as to verify the accuracy of our theoretical analysis.

The remainder of this paper is organized as follows. In Sect. 2, we give the fractional convection-diffusion equation (3) and its fully discrete finite volume element scheme. In Sect. 3, we analyze and prove the stability and convergence of the scheme. In Sect. 4, we give three numerical examples to verify the accuracy of the theoretical analysis. Finally in Sect. 5, we provide conclusions of this paper.

\section{Finite volume element scheme}

In this section, we consider the following two-dimensional space-fractional convectiondiffusion equation:

$$
\begin{cases}\frac{\partial u}{\partial t}-K_{1} \frac{\partial^{1+\beta} u}{\partial|x|^{1+\beta}}-K_{2} \frac{\partial^{1+\gamma} u}{\partial|y|^{1+\gamma}}+K_{3} \frac{\partial u}{\partial x}+K_{4} \frac{\partial u}{\partial y}=f, & (x, y) \in \Omega, 0<t \leq T, \\ u=0, & (x, y) \in \partial \Omega, 0<t \leq T, \\ u=u^{0}, & (x, y) \in \Omega, t=0,\end{cases}
$$

where $\Omega=\left(0, L_{1}\right) \times\left(0, L_{2}\right), 0<\beta, \gamma<1, \frac{\partial^{1+\beta} u}{\partial|x|^{1+\beta}}=K_{0, \beta} \frac{\partial}{\partial x}\left[\frac{\partial^{\beta} u}{\partial x^{\beta}}-\frac{\partial^{\beta} u}{\partial(-x)^{\beta}}\right], \frac{\partial^{1+\gamma} u}{\partial|y|^{1+\gamma}}=K_{0, \gamma} \frac{\partial}{\partial y}\left[\frac{\partial^{\gamma} u}{\partial y^{\gamma}}-\right.$ $\left.\frac{\partial^{\gamma} u}{\partial(-y)^{\gamma}}\right], K_{0, \beta}=-\frac{1}{2 \cos \left(\frac{(1+\beta) \pi}{2}\right)}>0, K_{0, \gamma}=-\frac{1}{2 \cos \left(\frac{(1+\gamma) \pi}{2}\right)}>0, \frac{\partial^{\beta} u}{\partial x^{\beta}}=\frac{1}{\Gamma(1-\beta)} \frac{\partial}{\partial x} \int_{0}^{x}(x-\xi)^{-\beta} u(\xi) d \xi$, $\frac{\partial^{\beta} u}{\partial(-x)^{\beta}}=-\frac{1}{\Gamma(1-\beta)} \frac{\partial}{\partial x} \int_{x}^{L_{1}}(\xi-x)^{-\beta} u(\xi) d \xi, K_{i}(i=1,2,3,4)$ are given constants, $K_{i} \geq 0(i=1,2)$, $K_{1}+K_{2}>0$, and $f \in L^{2}(\Omega)$. We assume that the solution of equation (3) has the smoothness and boundedness required in our analysis. 
Based on the definition of the Riesz fractional derivative, we can write the following equivalent form of equation (3):

$$
\left\{\begin{array}{rlrl}
\frac{\partial u}{\partial t} & \left.-K_{1} K_{0, \beta} \frac{\partial}{\partial x} \frac{\partial^{\beta} u}{\partial x^{\beta}}-\frac{\partial^{\beta} u}{\partial(-x)^{\beta}}\right]-K_{2} K_{0, \gamma} \frac{\partial}{\partial y}\left[\frac{\partial^{\gamma} u}{\partial y^{\gamma}}-\frac{\partial^{\gamma} u}{\partial(-y)^{\gamma}}\right] & \\
& +K_{3} \frac{\partial u}{\partial x}+K_{4} \frac{\partial u}{\partial y}=f, & & (x, y) \in \Omega, 0<t \leq T, \\
u=0, & & (x, y) \in \partial \Omega, 0<t \leq T, \\
u=u^{0}, & & (x, y) \in \Omega, t=0 .
\end{array}\right.
$$

Let $M$ be a positive integer, let $\tau=\frac{T}{M}$, and let $t_{m}=m \tau(m=0,1, \ldots, M)$. We use the $\mathrm{CN}$-scheme to discretize the time derivative in equation (3) and get

$$
\left\{\begin{array}{rlr}
u\left(x, y, t_{m}\right)-\frac{K_{0, \beta} K_{1} \tau}{2} \frac{\partial}{\partial x}\left(\frac{\partial^{\beta} u\left(x, y, t_{m}\right)}{\partial x^{\beta}}-\frac{\partial^{\beta} u\left(x, y, t_{m}\right)}{\partial(-x)^{\beta}}\right) & \\
& -\frac{K_{0, \gamma} K_{2} \tau}{2} \frac{\partial}{\partial y}\left(\frac{\partial^{\gamma} u\left(x, y, t_{m}\right)}{\partial y^{\gamma}}-\frac{\partial^{\gamma} u\left(x, y, t_{m}\right)}{\partial(-y)^{\gamma}}\right) & \\
& +\frac{\tau K_{3}}{2} \frac{\partial u\left(x, y, t_{m}\right)}{\partial x}+\frac{\tau K_{4}}{2} \frac{\partial u\left(x, y, t, t_{m}\right)}{\partial y} & \\
= & u\left(x, y, t_{m-1}\right)+\frac{K_{0, \beta} K_{1} \tau}{2} \frac{\partial}{\partial x}\left(\frac{\partial^{\beta} u\left(x, y, t_{m-1}\right)}{\partial x^{\beta}}-\frac{\partial^{\beta} u\left(x, y, t_{m-1}\right)}{\partial(-x)^{\beta}}\right) & \\
& +\frac{K_{0, \gamma} K_{2} \tau}{2} \frac{\partial}{\partial y}\left(\frac{\partial^{\gamma} u\left(x, y, t_{m-1}\right)}{\partial y^{\gamma}}-\frac{\partial^{\gamma} u\left(x, y, t_{m-1}\right)}{\partial(-y))^{\gamma}}\right)-\frac{\tau K_{3}}{2} \frac{\partial u\left(x, y, t_{m-1}\right)}{\partial x} & \\
& -\frac{\tau K_{4}}{2} \frac{\partial u\left(x, y, t_{m-1}\right)}{\partial y}+\tau f\left(x, y, t_{m-\frac{1}{2}}\right)+O\left(\tau^{3}\right), & (x, y) \in \Omega, 0<t \leq T \\
u=0, & (x, y) \in \partial \Omega, 0<t \leq T \\
u=u^{0}, & (x, y) \in \Omega, t=0
\end{array}\right.
$$

Next, let $N_{x}$ and $N_{y}$ be positive integers, and let $h_{x}=\frac{L_{1}}{N_{x}+1}$ and $h_{y}=\frac{L_{2}}{N_{y}+1}$. We divide the region $\Omega$ into a uniform grid with grid nodes $x_{i}=i h_{x}\left(0 \leq i \leq N_{x}+1\right)$ and $y_{j}=j h_{y}$ $\left(0 \leq j \leq N_{y}+1\right)$. Denote the element $\Omega_{i, j}=\left[x_{i}, x_{i+1}\right] \times\left[y_{j}, y_{j+1}\right]\left(0 \leq i \leq N_{x}, 0 \leq j \leq N_{y}\right)$. Besides, for $i=1, \ldots, N_{x}, j=1, \ldots, N_{y}, m=1, \ldots, M$, let $u_{i, j}^{m}$ be the finite volume element approximation of $u\left(x_{i}, y_{j}, t_{m}\right)$.

We take the trial function space $S_{h}(\Omega) \subset H_{0}^{1}(\Omega)$ as the linear element space with respect to the space subdivision mentioned above: for $u_{h} \in S_{h} \subset H_{0}^{1}(\Omega)$, we have $S_{h}=\left\{u_{h} \in C(\Omega)\right.$ : $\left.\left.u_{h}\right|_{\Omega_{i, j}} \in \mathcal{P}_{1} ;\left.u_{h}\right|_{\partial \Omega}=0\right\}$, where $\mathcal{P}_{1}$ is the set of all linear polynomials on $\Omega_{i, j}$. Then the approximate solution $u_{h}\left(x, y, t_{m}\right)$ of equation (3) can be expressed as follows:

$$
u_{h}\left(x, y, t_{m}\right)=\sum_{k=1}^{N_{x}} \sum_{l=1}^{N_{y}} u_{k, l}^{m} \phi_{k}^{x}(x) \phi_{l}^{y}(y),
$$

where $\phi_{k}^{x}(x)$ are linear basis functions in the direction of $x$, expressed as follows:

$$
\begin{aligned}
\phi_{0}^{x}(x) & = \begin{cases}\frac{x_{1}-x}{h}, & x \in\left[0, x_{1}\right], \\
0 & \text { elsewhere, }\end{cases} \\
\phi_{i}^{x}(x) & = \begin{cases}\frac{x-x_{i-1}}{h}, & x \in\left[x_{i-1}, x_{i}\right], \\
\frac{x_{i+1}-x}{h}, & x \in\left[x_{i}, x_{i+1}\right], 1 \leq i \leq N_{x}, \\
0 & \text { elsewhere, }\end{cases}
\end{aligned}
$$




$$
\phi_{N_{x}+1}^{x}(x)= \begin{cases}\frac{x-x_{N_{x}}}{h}, & x \in\left[x_{N_{x}}, L_{1}\right] \\ 0 & \text { elsewhere }\end{cases}
$$

and the definition of $\phi_{l}^{y}(y)$ in the $y$ direction is similar.

The dual elements are $\Omega_{0,0}^{*}=\left[x_{0}, x_{\frac{1}{2}}\right] \times\left[y_{0}, y_{\frac{1}{2}}\right], \Omega_{i, j}^{*}=\left[x_{i-\frac{1}{2}}, x_{i+\frac{1}{2}}\right] \times\left[y_{j-\frac{1}{2}}, y_{j+\frac{1}{2}}\right](1 \leq i \leq$ $\left.N_{x}, 1 \leq j \leq N_{y}\right)$, and $\Omega_{N_{x}+1, N_{y}+1}^{*}=\left[x_{N_{x}+\frac{1}{2}}, x_{N_{x}+1}\right] \times\left[y_{N_{y}+\frac{1}{2}}, y_{N_{y}+1}\right]$. Accordingly, we choose the test function space as the piecewise constant function space $V_{h}=\left\{v_{h} \in L^{2}(\Omega):\left.v_{h}\right|_{\Omega_{i, j}^{*}}=\right.$ constant; $\left.\left.v_{h}\right|_{\Omega_{0,0}^{*}}=0\right\}$.

The basis functions of $V_{h}$ are

$$
\psi_{i, j}(x, y)= \begin{cases}1, & (x, y) \in \Omega_{i, j}^{*}, 1 \leq i \leq N_{x}+1,1 \leq j \leq N_{y}+1, \\ 0 & \text { elsewhere. }\end{cases}
$$

On dual element $\Omega_{i, j}^{*}\left(1 \leq i \leq N_{x}+1,1 \leq j \leq N_{y}+1\right)$, we use $u_{h}^{m}=u_{h}\left(x, y, t_{m}\right)$ instead of $u\left(x, y, t_{m}\right)$ in equation (4) and omit the time-truncated error term. Then we get the following variational form:

$$
\begin{aligned}
\left(u_{h}^{m}, \psi_{i, j}\right) & -\left(\frac{K_{0, \beta} K_{1} \tau}{2} \frac{\partial}{\partial x}\left(\frac{\partial^{\beta} u_{h}^{m}}{\partial x^{\beta}}-\frac{\partial^{\beta} u_{h}^{m}}{\partial(-x)^{\beta}}\right), \psi_{i, j}\right) \\
& -\left(\frac{K_{0, \gamma} K_{2} \tau}{2} \frac{\partial}{\partial y}\left(\frac{\partial^{\gamma} u_{h}^{m}}{\partial y^{\gamma}}-\frac{\partial^{\gamma} u_{h}^{m}}{\partial(-y)^{\gamma}}\right), \psi_{i, j}\right) \\
& +\left(\frac{\tau K_{3}}{2} \frac{\partial u_{h}^{m}}{\partial x}, \psi_{i, j}\right)+\left(\frac{\tau K_{4}}{2} \frac{\partial u_{h}^{m}}{\partial y}, \psi_{i, j}\right) \\
= & \left(u_{h}^{m-1}, \psi_{i, j}\right)+\left(\frac{K_{0, \beta} K_{1} \tau}{2} \frac{\partial}{\partial x}\left(\frac{\partial^{\beta} u_{h}^{m-1}}{\partial x^{\beta}}-\frac{\partial^{\beta} u_{h}^{m-1}}{\partial(-x)^{\beta}}\right), \psi_{i, j}\right) \\
& +\left(\frac{K_{0, \gamma} K_{2} \tau}{2} \frac{\partial}{\partial y}\left(\frac{\partial^{\gamma} u_{h}^{m-1}}{\partial y^{\gamma}}-\frac{\partial^{\gamma} u_{h}^{m-1}}{\partial(-y)^{\gamma}}\right), \psi_{i, j}\right)-\left(\frac{\tau K_{3}}{2} \frac{\partial u_{h}^{m-1}}{\partial x}, \psi_{i, j}\right) \\
& -\left(\frac{\tau K_{4}}{2} \frac{\partial u_{h}^{m-1}}{\partial y}, \psi_{i, j}\right)+\tau\left(f^{m-\frac{1}{2}}, \psi_{i, j}\right) .
\end{aligned}
$$

We expand formula (8) to obtain the following fully discrete finite volume element scheme:

$$
\begin{aligned}
\sum_{k=1}^{N_{x}} \sum_{l=1}^{N_{y}} u_{k, l}^{m} \int_{x_{i-\frac{1}{2}}}^{x_{i+\frac{1}{2}}} \int_{y_{j-\frac{1}{2}}}^{y_{j+\frac{1}{2}}} \phi_{k}^{x}(x) \phi_{l}^{y}(y) d x d y & \\
& -\left.\frac{\tau K_{0, \beta} K_{1}}{2} \sum_{k=1}^{N_{x}} \sum_{l=1}^{N_{y}} u_{k, l}^{m}\left(\frac{\partial^{\beta} \phi_{k}^{x}(x)}{\partial x^{\beta}}-\frac{\partial^{\beta} \phi_{k}^{x}(x)}{\partial(-x)^{\beta}}\right)\right|_{x_{i-\frac{1}{2}}} ^{x_{i+\frac{1}{2}}} \int_{y_{j-\frac{1}{2}}}^{y_{j+\frac{1}{2}}} \phi_{l}^{y}(y) d y \\
& -\left.\frac{\tau K_{0, \gamma} K_{2}}{2} \sum_{k=1}^{N_{x}} \sum_{l=1}^{N_{y}} u_{k, l}^{m}\left(\frac{\partial^{\gamma} \phi_{l}^{y}(y)}{\partial y^{\gamma}}-\frac{\partial^{\gamma} \phi_{l}^{y}(y)}{\partial(-y)^{\gamma}}\right)\right|_{y_{j-\frac{1}{2}}} ^{y_{j+\frac{1}{2}}} \int_{x_{i-\frac{1}{2}}}^{x_{i+\frac{1}{2}}} \phi_{k}^{x}(x) d x \\
& +\left.\frac{\tau K_{3}}{2} \sum_{k=1}^{N_{x}} \sum_{l=1}^{N_{y}} u_{k, l}^{m} \phi_{k}^{x}(x)\right|_{x_{i-\frac{1}{2}}} ^{x_{i+\frac{1}{2}}} \int_{y_{j-\frac{1}{2}}}^{y_{j+\frac{1}{2}}} \phi_{l}^{y}(y) d y \\
& +\left.\frac{\tau K_{4}}{2} \sum_{k=1}^{N_{x}} \sum_{l=1}^{N_{y}} u_{k, l}^{m} \phi_{l}^{y}(y)\right|_{y_{j-\frac{1}{2}}} ^{y_{j+\frac{1}{2}}} \int_{x_{i-\frac{1}{2}}}^{x_{i+\frac{1}{2}}} \phi_{k}^{x}(x) d x
\end{aligned}
$$




$$
\begin{aligned}
= & \sum_{k=1}^{N_{x}} \sum_{l=1}^{N_{y}} u_{k, l}^{m-1} \int_{x_{i-\frac{1}{2}}}^{x_{i+\frac{1}{2}}} \int_{y_{j-\frac{1}{2}}}^{y_{j+\frac{1}{2}}} \phi_{k}^{x}(x) \phi_{l}^{y}(y) d x d y \\
& +\left.\frac{\tau K_{0, \beta} K_{1}}{2} \sum_{k=1}^{N_{x}} \sum_{l=1}^{N_{y}} u_{k, l}^{m-1}\left(\frac{\partial^{\beta} \phi_{k}^{x}(x)}{\partial x^{\beta}}-\frac{\partial^{\beta} \phi_{k}^{x}(x)}{\partial(-x)^{\beta}}\right)\right|_{x_{i-\frac{1}{2}}} ^{x_{i+\frac{1}{2}}} \int_{y_{j-\frac{1}{2}}}^{y_{j+\frac{1}{2}}} \phi_{l}^{y}(y) d y \\
& +\left.\frac{\tau K_{0, \gamma} K_{2}}{2} \sum_{k=1}^{N_{x}} \sum_{l=1}^{N_{y}} u_{k, l}^{m-1}\left(\frac{\partial^{\gamma} \phi_{l}^{y}(y)}{\partial y^{\gamma}}-\frac{\partial^{\gamma} \phi_{l}^{y}(y)}{\partial(-y)^{\gamma}}\right)\right|_{y_{j-\frac{1}{2}}} ^{y_{j+\frac{1}{2}}} \int_{x_{i-\frac{1}{2}}}^{x_{i+\frac{1}{2}}} \phi_{k}^{x}(x) d x \\
& -\left.\frac{\tau K_{3}}{2} \sum_{k=1}^{N_{x}} \sum_{l=1}^{N_{y}} u_{k, l}^{m-1} \phi_{k}^{x}(x)\right|_{x_{i-\frac{1}{2}}} ^{x_{i+\frac{1}{2}}} \int_{y_{j-\frac{1}{2}}}^{y_{j+\frac{1}{2}}} \phi_{l}^{y}(y) d y \\
& -\left.\frac{\tau K_{4}}{2} \sum_{k=1}^{N_{x}} \sum_{l=1}^{N_{y}} u_{k, l}^{m-1} \phi_{l}^{y}(y)\right|_{y_{j-\frac{1}{2}}} ^{y_{j+\frac{1}{2}}} \int_{x_{i-\frac{1}{2}}}^{x_{i+\frac{1}{2}}} \phi_{k}^{x}(x) d x \\
& +\tau \int_{x_{i-\frac{1}{2}}}^{x_{i+\frac{1}{2}}} \int_{y_{j-\frac{1}{2}}}^{y_{j+\frac{1}{2}}} f\left(x, y, t_{m-\frac{1}{2}}\right) d x d y .
\end{aligned}
$$

To facilitate the calculation, we introduce the following lemma.

Lemma 1 ([17]) For $k=1,2, \ldots, N_{x}$, we have

$$
\int_{x_{i-\frac{1}{2}}}^{x_{i+\frac{1}{2}}} \phi_{k}^{x}(x) d x=\frac{h_{x}}{8} \begin{cases}1, & |k-i|=1, \\ 6, & k=i, \\ 0 & \text { otherwise. }\end{cases}
$$

Lemma 2 ([17]) For $k=1,2, \ldots, N_{x}$, we have

$$
\begin{aligned}
& \left.\frac{\partial^{\beta} \phi_{k}^{x}(x)}{\partial x^{\beta}}\right|_{x_{i-\frac{1}{2}}}=\frac{1}{h_{x}^{\beta} \Gamma(2-\beta)} \begin{cases}0, & k>i, \\
s_{i-k}^{(1-\beta)}, & k \leq i,\end{cases} \\
& \left.\frac{\partial^{\beta} \phi_{k}^{x}(x)}{\partial x^{\beta}}\right|_{x_{i+\frac{1}{2}}}=\frac{1}{h_{x}^{\beta} \Gamma(2-\beta)} \begin{cases}0, & k>i+1, \\
s_{i-k+1}^{(1-\beta)}, & k \leq i+1,\end{cases} \\
& \left.\frac{\partial^{\beta} \phi_{k}^{x}(x)}{\partial(-x)^{\beta}}\right|_{x_{i-\frac{1}{2}}}=\frac{1}{h_{x}^{\beta} \Gamma(2-\beta)} \begin{cases}s_{i-k+1}^{(1-\beta)}, & k \geq i-1, \\
0, & k<i-1,\end{cases} \\
& \left.\frac{\partial^{\beta} \phi_{k}^{x}(x)}{\partial(-x)^{\beta}}\right|_{x_{i+\frac{1}{2}}}=\frac{1}{h_{x}^{\beta} \Gamma(2-\beta)} \begin{cases}s_{i-k}^{(1-\beta)}, & k \geq i, \\
0, & k<i,\end{cases}
\end{aligned}
$$

where

$$
s_{i}^{(1-\beta)}= \begin{cases}\left(\frac{1}{2}\right)^{(1-\beta)}, & i=0, \\ \left(\frac{3}{2}\right)^{(1-\beta)}-2 \cdot\left(\frac{1}{2}\right)^{(1-\beta)}, & i=1, \\ \left(i+\frac{1}{2}\right)^{(1-\beta)}-2 \cdot\left(i-\frac{1}{2}\right)^{(1-\beta)}+\left(i-\frac{3}{2}\right)^{(1-\beta)}, & 2 \leq i \leq N_{x} .\end{cases}
$$


Lemma 3 For $k=1,2, \ldots, N_{x}$, we have

$$
\phi_{k}^{x}(x)= \begin{cases}\frac{1}{2}, & k=i+1 \\ -\frac{1}{2}, & k=i-1 \\ 0 & \text { otherwise }\end{cases}
$$

Let $\mathbf{u}^{m}$ and $\mathbf{F}^{m}$ be $N:=N_{x} N_{y}$-dimensional vectors, defined as follows:

$$
\begin{aligned}
& \mathbf{u}^{m}=\left[u_{1,1}^{m}, \ldots, u_{N_{x}, 1}^{m}, u_{1,2}^{m}, \ldots, u_{N_{x}, 2}^{m}, \ldots, u_{1, N_{y}}^{m}, \ldots, u_{N_{x}, N_{y}}^{m},\right]^{T}, \\
& \mathbf{F}^{m}=\left[\bar{f}_{1,1}^{m}, \ldots, \bar{f}_{N_{x}, 1}^{m}, \bar{f}_{1,2}^{m}, \ldots, \bar{f}_{N_{x}, 2}^{m}, \ldots, \bar{f}_{1, N_{y}}^{m}, \ldots, \bar{f}_{N_{x}, N_{y}}^{m},\right]^{T},
\end{aligned}
$$

where

$$
\bar{f}_{i, j}^{m}:=\frac{1}{h_{x} h_{y}} \int_{x_{i-\frac{1}{2}}}^{x_{i+\frac{1}{2}}} \int_{y_{j-\frac{1}{2}}}^{y_{j+\frac{1}{2}}} f\left(x, y, t_{m-\frac{1}{2}}\right) d y d x .
$$

Then we define the following $N_{x}$ th-order mass matrix $\mathbf{A}_{x}, N_{x}$ th-order stiffness matrix $\mathbf{B}_{x}$, and $N_{x}$ th-order matrix $\mathbf{C}_{x}$ :

$$
\begin{aligned}
& \mathbf{A}_{x}=\frac{1}{8} \operatorname{tridiag}(1,6,1), \\
& \mathbf{B}_{x}=K_{0, \beta}\left[T^{1-\beta, N_{x}}+\left(T^{1-\beta, N_{x}}\right)^{T}\right], \\
& \mathbf{C}_{x}=\operatorname{tridiag}\left(-\frac{1}{2}, 0, \frac{1}{2}\right),
\end{aligned}
$$

where $T^{1-\beta, N_{x}}$ is a Toeplitz matrix of the following form:

$$
T^{1-\beta, N_{x}}=\left[\begin{array}{cccccc}
q_{1}^{(1-\beta)} & q_{0}^{(1-\beta)} & 0 & \cdots & 0 & 0 \\
q_{2}^{(1-\beta)} & q_{1}^{(1-\beta)} & q_{0}^{(1-\beta)} & \ddots & \ddots & 0 \\
\vdots & q_{2}^{(1-\beta)} & q_{1}^{(1-\beta)} & \ddots & \ddots & \vdots \\
\vdots & \ddots & \ddots & \ddots & \ddots & 0 \\
q_{N_{x}-1}^{(1-\beta)} & \ddots & \ddots & \ddots & q_{1}^{(1-\beta)} & q_{0}^{(1-\beta)} \\
q_{N_{x}}^{(1-\beta)} & q_{N_{x}-1}^{(1-\beta)} & \cdots & \cdots & q_{2}^{(1-\beta)} & q_{1}^{(1-\beta)}
\end{array}\right]
$$

with

$$
q_{i}^{(1-\beta)}= \begin{cases}-s_{0}^{(1-\beta)}, & i=0, \\ s_{i-1}^{(1-\beta)}-s_{i}^{(1-\beta)}, & 1 \leq i \leq N_{x} .\end{cases}
$$

Similarly, we can get three matrices $\mathbf{A}_{y}, \mathbf{B}_{y}$, and $\mathbf{C}_{y}$ in the $y$ direction defined as follows:

$$
\begin{aligned}
& \mathbf{A}_{y}=\frac{1}{8} \operatorname{tridiag}(1,6,1), \\
& \mathbf{B}_{y}=K_{0, \gamma}\left[T^{1-\gamma, N_{y}}+\left(T^{1-\gamma, N_{y}}\right)^{T}\right], \\
& \mathbf{C}_{y}=\operatorname{tridiag}\left(-\frac{1}{2}, 0, \frac{1}{2}\right) .
\end{aligned}
$$


We introduce the following definition and lemmas to give the matrix form of the fully discrete scheme (9).

Definition 1 ([41]) For $\mathbf{A} \in R^{m \times n}$ and $\mathbf{B} \in R^{r \times s}$, their Kronecker product is the partitioned $m r \times n s$ matrix

$$
\mathbf{A} \otimes \mathbf{B}=\left[\begin{array}{ccc}
a_{11} B & \cdots & a_{1 n} B \\
\vdots & \ddots & \vdots \\
a_{m 1} B & \cdots & a_{m n} B
\end{array}\right]
$$

Lemma 4 ([41]) The Kronecker product satisfies the following bilinear and associative properties:

$$
\begin{aligned}
& \mathbf{A} \otimes(\mathbf{B}+\mathbf{C})=\mathbf{A} \otimes \mathbf{B}+\mathbf{A} \otimes \mathbf{C} \quad \text { (if } B \text { and } C \text { have the same size), } \\
& (\mathbf{A}+\mathbf{C}) \otimes \mathbf{B}=\mathbf{A} \otimes \mathbf{B}+\mathbf{B} \otimes \mathbf{C} \quad(\text { if } A \text { and Bhave the same size }), \\
& (k \mathbf{A}) \otimes \mathbf{B}=\mathbf{A} \otimes(k \mathbf{B})=k(\mathbf{A} \otimes \mathbf{B}), \\
& (\mathbf{A} \otimes \mathbf{B}) \otimes \mathbf{C}=\mathbf{A} \otimes(\mathbf{B} \otimes \mathbf{C}),
\end{aligned}
$$

where $A, B$, and $C$ are matrices, and $k$ is a constant.

Lemma $5([17,41])$ If $A, B, C$, and $D$ are four matrices and if the matrix products $A C$ and $B D$ exist, then

\section{$(\mathbf{A} \otimes \mathbf{B})(\mathbf{C} \otimes \mathbf{D})=\mathbf{A C} \otimes \mathbf{B D}$.}

Lemma $\mathbf{6}([17,41])$ The matrix $\mathbf{A} \otimes \mathbf{B}$ is invertible if and only if $\mathbf{A}$ and $\mathbf{B}$ are invertible, and its inverse is $(\mathbf{A} \otimes \mathbf{B})^{T}=\mathbf{A}^{T} \otimes \mathbf{B}^{T}$.

Lemma $7([17,41])$ Let $\mathbf{A}$ and $\mathbf{B}$ be square matrices of sizes $n$ and $m$, respectively. Let $\lambda_{1}, \lambda_{2}, \ldots, \lambda_{n}$ be the eigenvalues of $\mathbf{A}$, and let $\mu_{1}, \mu_{2}, \ldots, \mu_{m}$ be the eigenvalues of $\mathbf{B}$. Then the eigenvalues of $\mathbf{A} \otimes \mathbf{B}$ are

$$
\lambda_{i} \mu_{j} \quad(i=1,2, \ldots, n, j=1,2, \ldots, m) .
$$

Thus the finite volume element scheme (9) can be expressed in the following matrix form:

$$
\begin{aligned}
\left(\mathbf{A}_{y}\right. & \left.\otimes \mathbf{A}_{x}+\eta_{\beta} \mathbf{A}_{y} \otimes \mathbf{B}_{x}+\eta_{\gamma} \mathbf{B}_{y} \otimes \mathbf{A}_{x}+\omega_{\beta} \mathbf{A}_{y} \otimes \mathbf{C}_{x}+\omega_{\gamma} \mathbf{C}_{y} \otimes \mathbf{A}_{x}\right) \mathbf{u}^{m} \\
\quad & \left(\mathbf{A}_{y} \otimes \mathbf{A}_{x}-\eta_{\beta} \mathbf{A}_{y} \otimes \mathbf{B}_{x}-\eta_{\gamma} \mathbf{B}_{y} \otimes \mathbf{A}_{x}-\omega_{\beta} \mathbf{A}_{y} \otimes \mathbf{C}_{x}-\omega_{\gamma} \mathbf{C}_{y} \otimes \mathbf{A}_{x}\right) \mathbf{u}^{m-1}+\tau \mathbf{F}^{m},
\end{aligned}
$$

where $\eta_{\beta}=\frac{K_{1} \tau}{2 \Gamma(2-\beta) h_{x}^{1+\beta}}, \eta_{\gamma}=\frac{K_{2} \tau}{2 \Gamma(2-\gamma) h_{y}^{1+\gamma}}$, and $\omega_{\beta}=\frac{K_{3} \tau}{2 h_{x}}, \omega_{\gamma}=\frac{K_{4} \tau}{2 h_{y}}$.

\section{Stability and convergence analysis}

In this section, to investigate the stability and convergence of scheme (22), we need the following lemmas. 
Lemma $8([17,42])$ An nth-order real matrix $\mathbf{C}$ is positive definite if and only if its symmetric part $\mathbf{H}=\frac{\mathbf{C}+\mathbf{C}^{T}}{2}$ is positive definite if and only if all eigenvalues of $\mathbf{H}$ are positive.

Lemma 9 ([17]) For any $0<\beta<1$, we have

$$
s_{1}^{(1-\beta)}<s_{0}^{(1-\beta)}, \quad 0<s_{2}^{(1-\beta)}-s_{1}^{(1-\beta)}+s_{0}^{(1-\beta)}<2,
$$

and for $i=2,3, \ldots$, we have

$$
s_{i}^{(1-\beta)}<s_{i+1}^{(1-\beta)}<0 .
$$

Lemma 10 ([17]) Suppose that $\mathbf{A}, \mathbf{B} \in R^{n \times n}$ are symmetric and positive definite. Then the matrix $\mathbf{A} \otimes \mathbf{B}$ is also symmetric and positive definite, that is,

$$
\mathbf{v}^{T}(\mathbf{A} \otimes \mathbf{B}) \mathbf{v}>0, \quad \mathbf{0} \neq v \in R^{n} .
$$

Lemma 11 ([17]) Let $\mathbf{A}_{x}$ and $\mathbf{A}_{y}$ be defined by (16) and (19), respectively. Then $\mathbf{A}_{y} \otimes \mathbf{A}_{x}$ is symmetric and positive definite, and for all $\mathbf{v} \in R^{N}$, we have

$$
\frac{1}{4} \mathbf{v}^{T} \mathbf{v} \leq \mathbf{v}^{T}\left(\mathbf{A}_{y} \otimes \mathbf{A}_{x}\right) \mathbf{v} \leq \mathbf{v}^{T} \mathbf{v} .
$$

Lemma 12 For $0<\beta, \gamma<1$, the stiffness matrices $\mathbf{A}_{y} \otimes \mathbf{B}_{x}$ and $\mathbf{B}_{y} \otimes \mathbf{A}_{x}$ are positive definite, that is,

$$
\mathbf{v}^{T}\left(\mathbf{A}_{y} \otimes \mathbf{B}_{x}\right) \mathbf{v}>0, \quad \mathbf{v}^{T}\left(\mathbf{B}_{y} \otimes \mathbf{A}_{x}\right) \mathbf{v}>0, \quad \mathbf{0} \neq v \in R^{N} .
$$

Proof According to Lemma 8, to prove that the matrices $\mathbf{A}_{y} \otimes \mathbf{B}_{x}$ and $\mathbf{B}_{y} \otimes \mathbf{A}_{x}$ are positive definite, we just have to prove that their symmetric parts

$$
\mathbf{G}_{1}:=\frac{\mathbf{A}_{y} \otimes \mathbf{B}_{x}+\left(\mathbf{A}_{y} \otimes \mathbf{B}_{x}\right)^{T}}{2}=\frac{\mathbf{A}_{y} \otimes \mathbf{B}_{x}+\mathbf{A}_{y} \otimes \mathbf{B}_{x}^{T}}{2}=\mathbf{A}_{y} \otimes \mathbf{G}_{x}
$$

and

$$
\mathbf{G}_{2}:=\frac{\mathbf{B}_{y} \otimes \mathbf{A}_{x}+\left(\mathbf{B}_{y} \otimes \mathbf{A}_{x}\right)^{T}}{2}=\frac{\mathbf{B}_{y} \otimes \mathbf{A}_{x}+\mathbf{B}_{y} \otimes \mathbf{A}_{x}^{T}}{2}=\mathbf{G}_{y} \otimes \mathbf{A}_{x}
$$

are positive definite, where $\mathbf{G}_{x}:=\frac{\mathbf{B}_{x}+\mathbf{B}_{x}^{T}}{2}, \mathbf{G}_{y}:=\frac{\mathbf{B}_{y}+\mathbf{B}_{y}^{T}}{2}$. According to (16) and (17), we find that the elements $g_{i, j}$ of $\mathbf{G}_{x}$ satisfy

$$
g_{i, j}=K_{0, \beta} \begin{cases}s_{i-j}^{(1-\beta)}-s_{i-j+1}^{(1-\beta)}, & j<i-1, \\ s_{1}^{(1-\beta)}-s_{2}^{(1-\beta)}-s_{0}^{(1-\beta)}, & j=i-1, \\ 2\left(s_{0}^{(1-\beta)}-s_{1}^{(1-\beta)}\right), & j=i, \\ g_{j, i}, & j \geq i+1 .\end{cases}
$$

By Lemma 9 we can infer that

$$
g_{i, i}>0, \quad g_{i, j}<0, \quad j \neq i,
$$


and

$$
\begin{aligned}
\sum_{j=1, j \neq i}^{N}\left|g_{i, j}\right|= & -\left[\sum_{j=1}^{i-2} g_{i, j}+g_{i, i-1}+g_{i, i+1}+\sum_{j=i+2}^{N} g_{i, j}\right] \\
= & 2 K_{0, \beta}\left(s_{2}^{(1-\beta)}-s_{1}^{(1-\beta)}+s_{0}^{(1-\beta)}\right)+K_{0, \beta} \sum_{j=2}^{i-1}\left(s_{j+1}^{(1-\beta)}-s_{j}^{(1-\beta)}\right) \\
& +K_{0, \beta} \sum_{j=2}^{N-i}\left(s_{j+1}^{(1-\beta)}-s_{j}^{(1-\beta)}\right) \\
< & 2 K_{0, \beta}\left(s_{2}^{(1-\beta)}-s_{1}^{(1-\beta)}+s_{0}^{(1-\beta)}+\sum_{j=2}^{N-1}\left(s_{j+1}^{(1-\beta)}-s_{j}^{(1-\beta)}\right)\right. \\
= & 2 K_{0, \beta}\left(s_{2}^{(1-\beta)}-s_{1}^{(1-\beta)}+s_{0}^{(1-\beta)}+s_{N}^{(1-\beta)}-s_{2}^{(1-\beta)}\right) \\
< & 2 K_{0, \beta}\left(s_{0}^{(1-\beta)}-s_{1}^{(1-\beta)}\right)=g_{i, i} .
\end{aligned}
$$

Thus the matrix $\mathbf{G}_{x}$ is a symmetric strictly diagonally dominant matrix with positive diagonal elements. It follows that the matrix $\mathbf{G}_{x}$ is positive definite. Similarly, we can prove that the matrix $\mathbf{G}_{y}$ is symmetrically positive definite. The matrices $\mathbf{A}_{x}$ and $\mathbf{A}_{y}$ are also symmetrically positive definite. Therefore by Lemma 10 we can infer that the matrices $\mathbf{G}_{1}$ and $\mathbf{G}_{2}$ are positive definite. This completes the proof.

Lemma 13 Let $\mathbf{C}_{x}$ and $\mathbf{C}_{y}$ be defined by (16) and (19), respectively. Then the matrices $\mathbf{C}_{y} \otimes \mathbf{A}_{x}$ and $\mathbf{A}_{y} \otimes \mathbf{C}_{x}$ satisfy

$$
\mathbf{v}^{T}\left(\mathbf{A}_{y} \otimes \mathbf{C}_{x}\right) \mathbf{v}=0, \quad \mathbf{v}^{T}\left(\mathbf{C}_{y} \otimes \mathbf{A}_{x}\right) \mathbf{v}=0, \quad \mathbf{0} \neq \mathbf{v} \in R^{N}
$$

Proof For any $N$-dimensional vector $\mathbf{v}=\left[v_{1}, v_{2}, \ldots, v_{N}\right]^{T}$, we write it as the $N_{y}$-dimensional block vector

$$
\mathbf{v}=\left[\mathbf{v}_{1}^{T}, \mathbf{v}_{2}^{T}, \ldots, \mathbf{v}_{N_{y}}^{T}\right]^{T},
$$

where each block $\mathbf{v}_{i}=\left[v_{(i-1) N_{x}+1}, v_{(i-1) N_{x}+2}, \ldots, v_{i N_{x}}\right]^{T}$ is an $N_{x}$-dimensional column vector. Because of the symmetry of $\mathbf{A}_{x}$, we get

$$
\begin{aligned}
\mathbf{v}^{T} & \left(\mathbf{C}_{y} \otimes \mathbf{A}_{x}\right) \mathbf{v} \\
& =\left[\mathbf{v}_{1}^{T}, \mathbf{v}_{2}^{T}, \ldots, \mathbf{v}_{N_{y}}^{T}\right] \operatorname{tridiag}\left(-\frac{1}{2} \mathbf{A}_{x}, 0, \frac{1}{2} \mathbf{A}_{x}\right)\left[\mathbf{v}_{1}^{T}, \mathbf{v}_{2}^{T}, \ldots, \mathbf{v}_{N_{y}}^{T}\right]^{T} \\
& =-\frac{1}{2} \sum_{i=1}^{N_{y}-1} \mathbf{v}_{i+1}^{T} \mathbf{A}_{x} \mathbf{v}_{i}+\frac{1}{2} \sum_{i=1}^{N_{y}-1} \mathbf{v}_{i}^{T} \mathbf{A}_{x} \mathbf{v}_{i+1} \\
& =0
\end{aligned}
$$


and since $\mathbf{C}_{x}$ is an antisymmetric matrix, we obtain

$$
\begin{aligned}
\mathbf{v}^{T} & \left(\mathbf{A}_{y} \otimes \mathbf{C}_{x}\right) \mathbf{v} \\
& =\frac{1}{8}\left[\mathbf{v}_{1}^{T}, \mathbf{v}_{2}^{T}, \ldots, \mathbf{v}_{N_{y}}^{T}\right] \operatorname{tridiag}\left(\mathbf{C}_{x}, 6 \mathbf{C}_{x}, \mathbf{C}_{x}\right)\left[\mathbf{v}_{1}^{T}, \mathbf{v}_{2}^{T}, \ldots, \mathbf{v}_{N_{y}}^{T}\right]^{T} \\
& =\frac{6}{8} \sum_{i=1}^{N_{y}-1} \mathbf{v}_{i}^{T} \mathbf{C}_{x} \mathbf{v}_{i}+\frac{1}{8} \sum_{i=1}^{N_{y}-1} \mathbf{v}_{i+1}^{T} \mathbf{C}_{x} \mathbf{v}_{i}+\frac{1}{8} \sum_{i=1}^{N_{y}-1} \mathbf{v}_{i}^{T} \mathbf{C}_{x} \mathbf{v}_{i+1} \\
& =0
\end{aligned}
$$

This completes the proof.

Next, according to Lemma 11, a two-dimensional weighted discrete norm is defined as

$$
\|\mathbf{v}\|_{A}:=\left(h_{x} h_{y} \mathbf{v}^{T}\left(\mathbf{A}_{y} \otimes \mathbf{A}_{x}\right) \mathbf{v}\right)^{\frac{1}{2}}
$$

and

$$
\left\|\mathbf{v}^{m}\right\|_{l^{2}}:=\left(h_{x} h_{y} \sum_{i=1}^{N_{x}} \sum_{j=1}^{N_{y}}\left(v_{i, j}^{m}\right)^{2}\right)^{\frac{1}{2}} .
$$

We can easily verify that $\|\cdot\|_{A}$ and $\|\cdot\|_{l^{2}}$ are equivalent, and by Lemma 11 we have

$$
\frac{1}{2}\|\mathbf{v}\|_{l^{2}} \leq\|\mathbf{v}\|_{A} \leq\|\mathbf{v}\|_{l^{2}}
$$

Theorem 1 For any $0<\beta, \gamma<1$, the finite volume element scheme (22) is uniquely solvable.

Proof It is obvious from equation (9) that the scheme is solvable. Next, to prove that scheme (22) is uniquely solvable, we need to prove that the homogeneous system of equations $\left(\mathbf{A}_{y} \otimes \mathbf{A}_{x}+\eta_{\beta} \mathbf{A}_{y} \otimes \mathbf{B}_{x}+\eta_{\gamma} \mathbf{B}_{y} \otimes \mathbf{A}_{x}+\omega_{\beta} \mathbf{A}_{y} \otimes \mathbf{C}_{x}+\omega_{\gamma} \mathbf{C}_{y} \otimes \mathbf{A}_{x}\right) \mathbf{u}^{m}=\mathbf{0}$ has only zero solution. Now left multiplying both sides of this system by $h_{x} h_{y}\left(\mathbf{u}^{m}\right)^{T}$, we organize it into the following form:

$$
\begin{aligned}
\left\|\mathbf{u}^{m}\right\|_{A}^{2}= & h_{x} h_{y}\left(\mathbf{u}^{m}\right)^{T}\left(\mathbf{A}_{y} \otimes \mathbf{A}_{x}\right) \mathbf{u}^{m} \\
= & -\eta_{\beta} h_{x} h_{y}\left(\mathbf{u}^{m}\right)^{T}\left(\mathbf{A}_{y} \otimes \mathbf{B}_{x}\right) \mathbf{u}^{m}-\eta_{\gamma} h_{x} h_{y}\left(\mathbf{u}^{m}\right)^{T}\left(\mathbf{B}_{y} \otimes \mathbf{A}_{x}\right) \mathbf{u}^{m} \\
& -\omega_{\beta} h_{x} h_{y}\left(\mathbf{u}^{m}\right)^{T}\left(\mathbf{A}_{y} \otimes \mathbf{C}_{x}\right) \mathbf{u}^{m}-\omega_{\gamma} h_{x} h_{y}\left(\mathbf{u}^{m}\right)^{T}\left(\mathbf{C}_{y} \otimes \mathbf{A}_{x}\right) \mathbf{u}^{m} .
\end{aligned}
$$

By Lemmas 12 and 13 we know

$$
\begin{aligned}
- & \eta_{\beta} h_{x} h_{y}\left(\mathbf{u}^{m}\right)^{T}\left(\mathbf{A}_{y} \otimes \mathbf{B}_{x}\right) \mathbf{u}^{m}-\eta_{\gamma} h_{x} h_{y}\left(\mathbf{u}^{m}\right)^{T}\left(\mathbf{B}_{y} \otimes \mathbf{A}_{x}\right) \mathbf{u}^{m} \\
& -\omega_{\beta} h_{x} h_{y}\left(\mathbf{u}^{m}\right)^{T}\left(\mathbf{A}_{y} \otimes \mathbf{C}_{x}\right) \mathbf{u}^{m}-\omega_{\gamma} h_{x} h_{y}\left(\mathbf{u}^{m}\right)^{T}\left(\mathbf{C}_{y} \otimes \mathbf{A}_{x}\right) \mathbf{u}^{m} \leq 0,
\end{aligned}
$$

so $\left\|\mathbf{u}^{m}\right\|_{A}^{2} \leq 0$, that is, $\left\|\mathbf{u}^{m}\right\|_{A}^{2}=0$, and thus $\mathbf{u}^{m}=\mathbf{0}$. This completes the proof. 
Theorem 2 For any $0<\beta, \gamma<1$, the finite volume element scheme (22) is unconditionally stable in the sense of the discrete norm, that is,

$$
\left\|\mathbf{u}^{m}\right\|_{l^{2}} \leq 2\left\|\mathbf{u}^{0}\right\|_{l^{2}}+4 \tau \sum_{d=1}^{m}\left\|\mathbf{F}^{d}\right\|_{l^{2}}, \quad 1 \leq m \leq M .
$$

Proof First, we organize (22) into the following form:

$$
\begin{aligned}
& \left(\mathbf{A}_{y} \otimes \mathbf{A}_{x}\right)\left(\mathbf{u}^{m}-\mathbf{u}^{m-1}\right)+\eta_{\beta}\left(\mathbf{A}_{y} \otimes \mathbf{B}_{x}\right)\left(\mathbf{u}^{m}+\mathbf{u}^{m-1}\right)+\eta_{\gamma}\left(\mathbf{B}_{y} \otimes \mathbf{A}_{x}\right)\left(\mathbf{u}^{m}+\mathbf{u}^{m-1}\right) \\
& +\omega_{\beta}\left(\mathbf{A}_{y} \otimes \mathbf{C}_{x}\right)\left(\mathbf{u}^{m}+\mathbf{u}^{m-1}\right)+\omega_{\gamma}\left(\mathbf{C}_{y} \otimes \mathbf{A}_{x}\right)\left(\mathbf{u}^{m}+\mathbf{u}^{m-1}\right)=\tau \mathbf{F}^{m} .
\end{aligned}
$$

Then left multiplying both sides of equation (37) by $h_{x} h_{y}\left(\mathbf{u}^{m}+\mathbf{u}^{m-1}\right)^{T}$, by Lemmas 11,12 , and 13 we get

$$
\begin{aligned}
\left\|\mathbf{u}^{m}\right\|_{A}^{2}-\left\|\mathbf{u}^{m-1}\right\|_{A}^{2} & =h_{x} h_{y}\left(\mathbf{u}^{m}+\mathbf{u}^{m-1}\right)^{T}\left(\mathbf{A}_{y} \otimes \mathbf{A}_{x}\right)\left(\mathbf{u}^{m}-\mathbf{u}^{m-1}\right) \\
& \leq \tau h_{x} h_{y}\left(\mathbf{u}^{m}+\mathbf{u}^{m-1}\right)^{T} \mathbf{F}^{m} \\
& \leq \tau\left\|\mathbf{u}^{m}+\mathbf{u}^{m-1}\right\|_{l^{2}}\left\|\mathbf{F}^{m}\right\|_{l^{2}} \\
& \leq 2 \tau\left\|\mathbf{u}^{m}+\mathbf{u}^{m-1}\right\|_{A}\left\|\mathbf{F}^{m}\right\|_{l^{2}},
\end{aligned}
$$

where in the last two steps we used the Cauchy-Schwarz inequality and formula (33), respectively. Subtracting $\left\|\mathbf{u}^{m}+\mathbf{u}^{m-1}\right\|_{A}$ from both sides of this equation, we have

$$
\left\|\mathbf{u}^{m}\right\|_{A} \leq\left\|\mathbf{u}^{m-1}\right\|_{A}+2 \tau\left\|\mathbf{F}^{m}\right\|_{l^{2}} .
$$

By iteration we get

$$
\left\|\mathbf{u}^{m}\right\|_{A} \leq\left\|\mathbf{u}^{0}\right\|_{A}+2 \tau \sum_{d=1}^{m}\left\|\mathbf{F}^{d}\right\|_{l^{2}}
$$

Again by formula (33) we obtain

$$
\left\|\mathbf{u}^{m}\right\|_{l^{2}} \leq 2\left\|\mathbf{u}^{0}\right\|_{l^{2}}+4 \tau \sum_{d=1}^{m}\left\|\mathbf{F}^{d}\right\|_{l^{2}} .
$$

This completes the proof.

Lemma $14([15])$ For $W(x) \in C^{2}[0, L]$, we have

$$
W(x)=\sum_{k=1}^{N_{x}} W_{k} \phi_{k}^{x}(x)+O\left(h^{2}\right)
$$

and

$$
\int_{x_{i-\frac{1}{2}}}^{x_{i+\frac{1}{2}}} W(x) d x=\int_{x_{i-\frac{1}{2}}}^{x_{i+\frac{1}{2}}} \sum_{k=1}^{N_{x}} W_{k} \phi_{k}^{x}(x) d x+O\left(h^{3}\right) .
$$


Lemma 15 ([15]) Let $W(x) \in C^{\beta+1}[0, L]$ for some $0<\beta<1$. Then

$$
\begin{aligned}
& \left.\left(\frac{\partial^{\beta} W\left(x+\frac{h}{2}\right)}{\partial x^{\beta}}-\frac{\partial^{\beta} W\left(x-\frac{h}{2}\right)}{\partial x^{\beta}}\right)\right|_{x_{i}}=\left.\frac{\partial^{\beta} W(x)}{\partial x^{\beta}}\right|_{x_{i-\frac{1}{2}}} ^{x_{i+\frac{1}{2}}}=O(h), \\
& \left.\left(\frac{\partial^{\beta} W\left(x+\frac{h}{2}\right)}{\partial(-x)^{\beta}}-\frac{\partial^{\beta} W\left(x-\frac{h}{2}\right)}{\partial(-x)^{\beta}}\right)\right|_{x_{i}}=\left.\frac{\partial^{\beta} W(x)}{\partial(-x)^{\beta}}\right|_{x_{i-\frac{1}{2}}} ^{x_{i+\frac{1}{2}}}=O(h) .
\end{aligned}
$$

Corollary 1 ([15]) Combining Lemmas 14 and 15, we get

$$
\begin{aligned}
& \left.\frac{\partial^{\beta} u(x)}{\partial x^{\beta}}\right|_{x_{i-\frac{1}{2}}} ^{x_{i+\frac{1}{2}}}=\sum_{k=1}^{N_{x}} u_{k}\left(\frac{\partial^{\beta} \phi_{k}^{x}\left(x_{i+\frac{h}{2}}\right)}{\partial x^{\beta}}-\frac{\partial^{\beta} \phi_{k}^{x}\left(x_{i-\frac{h}{2}}\right)}{\partial x^{\beta}}\right)+O\left(h^{3}\right), \\
& \left.\frac{\partial^{\beta} u(x)}{\partial(-x)^{\beta}}\right|_{x_{i-\frac{1}{2}}} ^{x_{i+\frac{1}{2}}}=\sum_{k=1}^{N_{x}} u_{k}\left(\frac{\partial^{\beta} \phi_{k}^{x}\left(x_{i+\frac{h}{2}}\right)}{\partial(-x)^{\beta}}-\frac{\partial^{\beta} \phi_{k}^{x}\left(x_{i-\frac{h}{2}}\right)}{\partial(-x)^{\beta}}\right)+O\left(h^{3}\right) .
\end{aligned}
$$

Lemma 16 For $W(x) \in C^{1}[0, L]$, we have

$$
\left.\left[W\left(x+\frac{h}{2}\right)-W\left(x-\frac{h}{2}\right)\right]\right|_{x_{i}}=\left.W(x)\right|_{x_{i}-\frac{1}{2}} ^{x_{i}+\frac{1}{2}}=O(h) .
$$

Proof Applying the Lagrange mean value theorem, we get

$$
W\left(x+\frac{h}{2}\right)-W\left(x-\frac{h}{2}\right)=h W^{\prime}(\xi)=h \frac{\partial W(\xi)}{\partial x}, \quad x-\frac{h}{2}<\xi<x+\frac{h}{2},
$$

Since $W(x) \in C^{1}[0, L]$, we have

$$
\left|W\left(x+\frac{h}{2}\right)-W\left(x-\frac{h}{2}\right)\right|=\left|h \frac{\partial W(\xi)}{\partial x}\right| \leq C h .
$$

This completes this proof.

Corollary 2 Combining Lemmas 14 and 16, we obtain

$$
\left.u(x)\right|_{x_{i}-\frac{1}{2}} ^{x_{i}+\frac{1}{2}}=\sum_{k=1}^{N_{x}} u_{k}\left[\phi_{k}^{x}\left(x_{i}+\frac{1}{2}\right)-\phi_{k}^{x}\left(x_{i}-\frac{1}{2}\right)\right]+O\left(h^{3}\right) .
$$

Proof By Lemma 14 we have

$$
u(x)=\sum_{k=1}^{N_{x}} u_{k} \phi_{k}^{x}(x)+O\left(h^{2}\right),
$$

and by Lemma 16 we get

$$
\left[u(x)-\sum_{k=1}^{N_{x}} u_{k} \phi_{k}^{x}(x)\right]_{x_{i}-\frac{1}{2}}^{x_{i}+\frac{1}{2}}=O\left(h \cdot h^{2}\right)=O\left(h^{3}\right) .
$$

This completes this proof. 
Theorem 3 Let $\mathbf{U}^{m} \in H^{3}(\Omega)$, and let $U_{i, j}^{m}=u\left(x_{i}, y_{j}, t_{m}\right)$ be the exact solution of equation (3), let $u_{i, j}^{m}=u\left(x_{i}, y_{j}, t_{m}\right)$ be the numerical solution of the finite volume element scheme (22). Then we have the error estimate

$$
\left\|\mathbf{U}^{m}-\mathbf{u}^{m}\right\|_{l^{2}} \leq 4 T \sqrt{L_{1} L_{2}} C\left(\tau^{2}+h_{x}^{2}+h_{y}^{2}\right) .
$$

Proof By the previous analysis the exact solution $U_{i, j}^{m}=u\left(x_{i}, y_{j}, t_{m}\right)$ of the form (22) has a local truncation error

$$
O\left(\tau^{3} h_{x} h_{y}+\tau h_{x}^{3} h_{y}+\tau h_{x} h_{y}^{3}\right)=\tau O\left(\tau^{2} h_{x} h_{y}+h_{x}^{3} h_{y}+h_{x} h_{y}^{3}\right)
$$

Let $\mathbf{e}^{m}=\mathbf{U}^{m}-\mathbf{u}^{m}$ be the global truncation error of the initial value $\mathbf{e}^{0}=\mathbf{0}$. We can obtain the following error equation in the matrix form:

$$
\begin{aligned}
\left(\mathbf{A}_{y} \otimes \mathbf{A}_{x}+\eta_{\beta} \mathbf{A}_{y} \otimes \mathbf{B}_{x}+\eta_{\gamma} \mathbf{B}_{y} \otimes \mathbf{A}_{x}+\omega_{\beta} \mathbf{A}_{y} \otimes \mathbf{C}_{x}+\omega_{\gamma} \mathbf{C}_{y} \otimes \mathbf{A}_{x}\right) \mathbf{e}^{m} \\
=\left(\mathbf{A}_{y} \otimes \mathbf{A}_{x}-\eta_{\beta} \mathbf{A}_{y} \otimes \mathbf{B}_{x}-\eta_{\gamma} \mathbf{B}_{y} \otimes \mathbf{A}_{x}-\omega_{\beta} \mathbf{A}_{y} \otimes \mathbf{C}_{x}-\omega_{\gamma} \mathbf{C}_{y} \otimes \mathbf{A}_{x}\right) \mathbf{e}^{m-1} \\
\quad+\tau O\left(\tau^{2}+h_{x}^{2}+h_{y}^{2}\right) \mathbf{E},
\end{aligned}
$$

where $\mathbf{E}=[1,1, \ldots, 1]^{T}$. Then according to the statement on stability in Theorem 2 , we have

$$
\begin{aligned}
\left\|\mathbf{e}^{m}\right\|_{l^{2}} & \leq 2\left\|\mathbf{e}^{0}\right\|_{l^{2}}+4 \tau \sum_{d=1}^{m}\left\|C\left(\tau^{2}+h_{x}^{2}+h_{y}^{2}\right) \mathbf{E}^{d}\right\|_{l^{2}} \\
& \leq 4 T \sqrt{L_{1} L_{2}} C\left(\tau^{2}+h_{x}^{2}+h_{y}^{2}\right) .
\end{aligned}
$$

This completes this proof.

\section{Numerical examples}

First, we give a fast algorithm for calculating the product of block Toeplitz matrices with vectors [21, 43]. It is known that thematrices $\mathbf{A}_{y} \otimes \mathbf{A}_{x}, \mathbf{A}_{y} \otimes \mathbf{B}_{x}, \mathbf{B}_{y} \otimes \mathbf{A}_{x}, \mathbf{A}_{y} \otimes \mathbf{C}_{x}$, and $\mathbf{C}_{y} \otimes \mathbf{A}_{x}$ are block Toeplitz-Toeplitz block matrices. Taking the matrix $\mathbf{A}_{y} \otimes \mathbf{A}_{x}$ as an example, we first extend every $N_{x} \times N_{x}$ Toeplitz block matrix of $\mathbf{A}_{y} \otimes \mathbf{A}_{x}$ into a $2 N_{x} \times 2 N_{x}$ cyclic matrix, so that the original matrix is expanded into an $N_{y} \times N_{y}$ block Toeplitzcyclic block matrix. Then we assemble the new block Toeplitz matrix into a $2 N_{y} \times 2 N_{y}$ block cycle matrix, so that we generate a $2 N_{y} \times 2 N_{y}$ block cycle matrix with each inner block of $2 N_{x} \times 2 N_{x}$ block cycle, represented by $C$. Let $F=F_{2 N_{x}} \otimes F_{2 N_{y}}$ represent the twodimensional discrete Fourier transform matrix, and let $c$ represent the first column of the newly assembled block cyclic-cyclic block matrix, so that we obtain the Fourier transform of the vector $c$ :

$$
\hat{c}=F c=\left(F_{2 N_{x}} \otimes F_{2 N_{y}}\right) c .
$$

Next, we use the following property of the cyclic matrix:

$$
C=F^{-1} \operatorname{diag}(\hat{c}) F=\left(F_{2 N_{x}} \otimes F_{2 N_{y}}\right)^{-1} \operatorname{diag}(\hat{c})\left(F_{2 N_{x}} \otimes F_{2 N_{y}}\right) .
$$


We can implement the fast matrix multiplication vector algorithm for two-dimensional problems, which reduces the computation amount of matrix vector multiplication from the traditional $O\left(N^{3}\right)$ to $O(N \log N)$.

We give several numerical examples to verify the validity of the finite volume element scheme. Just for simplicity of the rest of calculation, let $h_{x}=h_{y}=h$.

Example 1 First, we consider the following two-dimensional Riesz fractional diffusion equation:

$$
\begin{cases}\frac{\partial u}{\partial t}-\frac{\partial^{1+\beta} u}{\partial|x|^{1+\beta}}-\frac{\partial^{1+\gamma} u}{\partial|y|^{1+\gamma}}=f, & (x, y) \in \Omega, 0<t \leq 1, \\ u=0, & (x, y) \in \partial \Omega, 0<t \leq 1, \\ u=x^{2}(1-x)^{2} y^{2}(1-y)^{2}, & (x, y) \in \Omega, t=0,\end{cases}
$$

where $\Omega=(0,1) \times(0,1), 0<\beta, \gamma<1$, the source term $f=e^{t} x^{2}(1-x)^{2} y^{2}(1-y)^{2}+$ $\frac{e^{t}}{2 \cos \left(\frac{(1+\beta) \pi}{2}\right)}\left\{\frac{24}{\Gamma(4-\beta)}\left[x^{3-\beta}+(1-x)^{3-\beta}\right]-\frac{12}{\Gamma(3-\beta)}\left[x^{2-\beta}+(1-x)^{2-\beta}\right]+\frac{2}{\Gamma(2-\beta)}\left[x^{1-\beta}+(1-x)^{1-\beta}\right]\right\} y^{2}(1-$ $y)^{2}+\frac{e^{t}}{2 \cos \left(\frac{(1+\gamma) \pi}{2}\right)}\left\{\frac{24}{\Gamma(4-\gamma)}\left[y^{3-\gamma}+(1-y)^{3-\gamma}\right]-\frac{12}{\Gamma(3-\gamma)}\left[y^{2-\gamma}+(1-y)^{2-\gamma}\right]+\frac{2}{\Gamma(2-\gamma)}\left[y^{1-\gamma}+(1-\right.\right.$ $\left.\left.y)^{1-\gamma}\right]\right\} x^{2}(1-x)^{2}$, and the exact solution $u=e^{t} x^{2}(1-x)^{2} y^{2}(1-y)^{2}$.

We define the $L^{2}$-norm of the error between the exact solution $\mathbf{U}^{M}$ and numerical solution $\mathbf{u}^{M}$ as follows:

$$
\text { Error }=\left\|\mathbf{U}^{M}-\mathbf{u}^{M}\right\|=\sqrt{h^{2} \sum_{i=1}^{N}\left[U_{i}^{M}-u_{i}^{M}\right]^{2}} .
$$

Based on the above analysis, the $L^{2}$-norm and convergence order of Example 1 calculated by MATLAB program are shown in the following tables. Tables 1 and 2 give the $L^{2}$-norm and spatial convergence rate under the condition of $\tau=h$ and different $\beta, \gamma$, Tables 3 and 4 give the $L^{2}$-norm and time convergence rate under the condition of $h=2^{-8}$ and different $\beta, \gamma$. In agreement with the theoretical analysis given before, the time convergence rate and the space convergence rate are both of order 2 . Tables 5 and 6 show the comparison of the calculation time of the three different calculation methods. We easily see that the fast bicgstab method greatly improves the calculation speed.

To make the data results more intuitive, we present two groups of images. Figure 1 shows the comparison of numerical solutions and exact solutions under the same conditions. We can find that the two groups of images are roughly the same. Figure 2 shows the spatial convergence order images under different $\beta$ and $\gamma$ conditions, and we clearly see that the image data conform to the theoretical results.

\begin{tabular}{|c|c|c|c|c|c|c|}
\hline \multirow[t]{2}{*}{$\bar{h}$} & \multicolumn{2}{|l|}{$\beta=\gamma=0.3$} & \multicolumn{2}{|l|}{$\beta=\gamma=0.5$} & \multicolumn{2}{|l|}{$\beta=\gamma=0.8$} \\
\hline & Error & Rate & Error & Rate & Error & Rate \\
\hline $1 / 2^{4}$ & $3.7106 e-05$ & - & $3.7573 e-05$ & - & $3.9845 \mathrm{e}-05$ & - \\
\hline $1 / 2^{5}$ & $1.0136 \mathrm{e}-05$ & 1.8722 & $1.0213 \mathrm{e}-05$ & 1.8793 & $1.0749 \mathrm{e}-05$ & 1.8902 \\
\hline $1 / 2^{6}$ & $2.6712 \mathrm{e}-06$ & 1.9239 & $2.6777 \mathrm{e}-06$ & 1.9313 & $2.8043 \mathrm{e}-06$ & 1.9385 \\
\hline $1 / 2^{7}$ & $6.9103 e-07$ & 1.9507 & $6.8902 \mathrm{e}-07$ & 1.9584 & $7.1849 \mathrm{e}-07$ & 1.9646 \\
\hline $1 / 2^{8}$ & $1.7735 \mathrm{e}-07$ & 1.9621 & $1.7567 \mathrm{e}-07$ & 1.9717 & $1.8242 \mathrm{e}-07$ & 1.9777 \\
\hline
\end{tabular}

Table $1 L^{2}$-norm and convergence rate when $\beta=\gamma=0.3,0.5,0.8$ and $\tau=h$ 
Table $2 L^{2}$-norm and convergence rate when $\beta=0.3,0.4,0.5, \gamma=0.8,0.7,0.6$, and $\tau=h$

\begin{tabular}{|c|c|c|c|c|c|c|}
\hline \multirow[t]{2}{*}{$h$} & \multicolumn{2}{|c|}{$\beta=0.3, \gamma=0.8$} & \multicolumn{2}{|c|}{$\beta=0.4, \gamma=0.7$} & \multicolumn{2}{|c|}{$\beta=0.5, \gamma=0.6$} \\
\hline & Error & Rate & Error & Rate & Error & Rate \\
\hline $1 / 2^{4}$ & $3.9727 \mathrm{e}-05$ & - & $3.8506 \mathrm{e}-05$ & - & $3.7885 \mathrm{e}-05$ & - \\
\hline $1 / 2^{5}$ & $1.0777 \mathrm{e}-05$ & 1.8822 & $1.0454 \mathrm{e}-05$ & 1.8810 & $1.0287 \mathrm{e}-05$ & 1.8808 \\
\hline $1 / 2^{6}$ & $2.8237 \mathrm{e}-06$ & 1.9323 & $2.7390 \mathrm{e}-06$ & 1.9323 & $2.6944 \mathrm{e}-06$ & 1.9328 \\
\hline $1 / 2^{7}$ & $7.2630 \mathrm{e}-07$ & 1.9589 & $7.0428 \mathrm{e}-07$ & 1.9594 & $6.9254 \mathrm{e}-07$ & 1.9600 \\
\hline $1 / 2^{8}$ & $1.8508 \mathrm{e}-07$ & 1.9724 & $1.7935 \mathrm{e}-07$ & 1.9734 & $1.7631 \mathrm{e}-07$ & 1.9738 \\
\hline
\end{tabular}

Table $3 L^{2}$-norm and convergence rate when $\beta=\gamma=0.3,0.5,0.8$ and $h=2^{-8}$

\begin{tabular}{|c|c|c|c|c|c|c|}
\hline \multirow[t]{2}{*}{$\tau$} & \multicolumn{2}{|l|}{$\beta=\gamma=0.3$} & \multicolumn{2}{|l|}{$\beta=\gamma=0.5$} & \multicolumn{2}{|l|}{$\beta=\gamma=0.8$} \\
\hline & Error & Rate & Error & Rate & Error & Rate \\
\hline $1 / 2^{1}$ & $1.1622 \mathrm{e}-04$ & - & $1.1477 \mathrm{e}-04$ & - & $1.1043 \mathrm{e}-04$ & - \\
\hline $1 / 2^{2}$ & $3.0637 \mathrm{e}-05$ & 1.9235 & $3.1111 \mathrm{e}-05$ & 1.8832 & $3.1833 e-05$ & 1.7945 \\
\hline $1 / 2^{3}$ & $7.5963 \mathrm{e}-06$ & 2.0119 & $7.7286 \mathrm{e}-06$ & 2.0091 & 7.9457e-06 & 2.0023 \\
\hline $1 / 2^{4}$ & $1.7927 \mathrm{e}-06$ & 2.0832 & $1.8160 \mathrm{e}-06$ & 2.0894 & $1.8646 \mathrm{e}-06$ & 2.0931 \\
\hline
\end{tabular}

Table $4 L^{2}$-norm and convergence rate when $\beta=0.3,0.4,0.5, \gamma=0.8,0.7,0.6$, and $h=2^{-8}$

\begin{tabular}{|c|c|c|c|c|c|c|}
\hline \multirow[t]{2}{*}{$\tau$} & \multicolumn{2}{|c|}{$\beta=0.3, \gamma=0.1$} & \multicolumn{2}{|c|}{$\beta=0.4, \gamma=0.7$} & \multicolumn{2}{|c|}{$\beta=0.5, \gamma=0.6$} \\
\hline & Error & Rate & Error & Rate & Error & Rate \\
\hline $1 / 2^{1}$ & $1.1640 \mathrm{e}-04$ & - & $1.1388 \mathrm{e}-04$ & - & $1.1415 \mathrm{e}-04$ & - \\
\hline $1 / 2^{2}$ & $3.0373 e-05$ & 1.9382 & $3.1248 \mathrm{e}-05$ & 1.8657 & $3.1216 \mathrm{e}-05$ & 1.8706 \\
\hline $1 / 2^{3}$ & $7.5240 \mathrm{e}-06$ & 2.0132 & $7.7430 \mathrm{e}-06$ & 2.0128 & $7.7576 \mathrm{e}-06$ & 2.0086 \\
\hline $1 / 2^{4}$ & $1.7773 \mathrm{e}-06$ & 2.0818 & $1.8122 \mathrm{e}-06$ & 2.0952 & $1.8198 \mathrm{e}-06$ & 2.0918 \\
\hline
\end{tabular}

Table 5 Comparison of the time taken by Gauss elimination, BICGSTAB, and FAST-BICGSTAB methods when $\beta=\gamma=0.3$ and $\tau=h$

\begin{tabular}{|c|c|c|c|c|c|c|}
\hline h & Method & Time 1 & Method & Time 2 & Method & Time 3 \\
\hline $1 / 2^{4}$ & Gauss & $0.009686 \mathrm{~s}$ & BICGSTAB & $0.008474 \mathrm{~s}$ & FAST-BICGSTAB & $0.061735 \mathrm{~s}$ \\
\hline $1 / 2^{5}$ & & $0.526368 \mathrm{~s}$ & & $0.241088 \mathrm{~s}$ & & $0.354841 \mathrm{~s}$ \\
\hline $1 / 2^{6}$ & & $61.452817 \mathrm{~s}$ & & $6.339914 \mathrm{~s}$ & & $2.628164 \mathrm{~s}$ \\
\hline $1 / 2^{7}$ & & $4160.579938 \mathrm{~s}$ & & $183.270371 \mathrm{~s}$ & & $15.732230 \mathrm{~s}$ \\
\hline $1 / 2^{8}$ & & - & & - & & $182.436328 \mathrm{~s}$ \\
\hline $1 / 2^{9}$ & & - & & - & & $1897.905645 \mathrm{~s}$ \\
\hline
\end{tabular}

Table 6 Comparison of the time taken by Gauss elimination, BICGSTAB, and FAST-BICGSTAB methods when $\beta=\gamma=0.8$ and $\tau=h$

\begin{tabular}{|c|c|c|c|c|c|c|}
\hline $\bar{h}$ & Method & Time 1 & Method & Time 2 & Method & Time 3 \\
\hline$\overline{1 / 2^{4}}$ & Gauss & $0.009587 \mathrm{~s}$ & BICGSTAB & $0.012225 \mathrm{~s}$ & FAST-BICGSTAB & $0.123099 \mathrm{~s}$ \\
\hline $1 / 2^{5}$ & & $0.500738 s$ & & $0.503436 \mathrm{~s}$ & & $0.947818 s$ \\
\hline $1 / 2^{6}$ & & $62.099249 \mathrm{~s}$ & & $24.926117 \mathrm{~s}$ & & $9.177119 \mathrm{~s}$ \\
\hline $1 / 2^{7}$ & & $4200.554038 \mathrm{~s}$ & & $995.119451 \mathrm{~s}$ & & $67.557581 \mathrm{~s}$ \\
\hline $1 / 2^{8}$ & & - & & - & & $962.377248 \mathrm{~s}$ \\
\hline $1 / 2^{9}$ & & - & & - & & $10141.873806 \mathrm{~s}$ \\
\hline
\end{tabular}

Example 2 Consider the following two-dimensional Riesz fractional convection-diffusion equation:

$$
\begin{cases}\frac{\partial u}{\partial t}-\frac{\partial^{1+\beta} u}{\partial|x|^{1+\beta}}-\frac{\partial^{1+\gamma} u}{\partial|y|^{1+\gamma}}+\frac{\partial u}{\partial x}+\frac{\partial u}{\partial y}=f, & (x, y) \in \Omega, 0<t \leq 1, \\ u=0, & (x, y) \in \partial \Omega, 0<t \leq 1, \\ u=x^{2}(1-x)^{2} y^{2}(1-y)^{2}, & (x, y) \in \Omega, t=0\end{cases}
$$



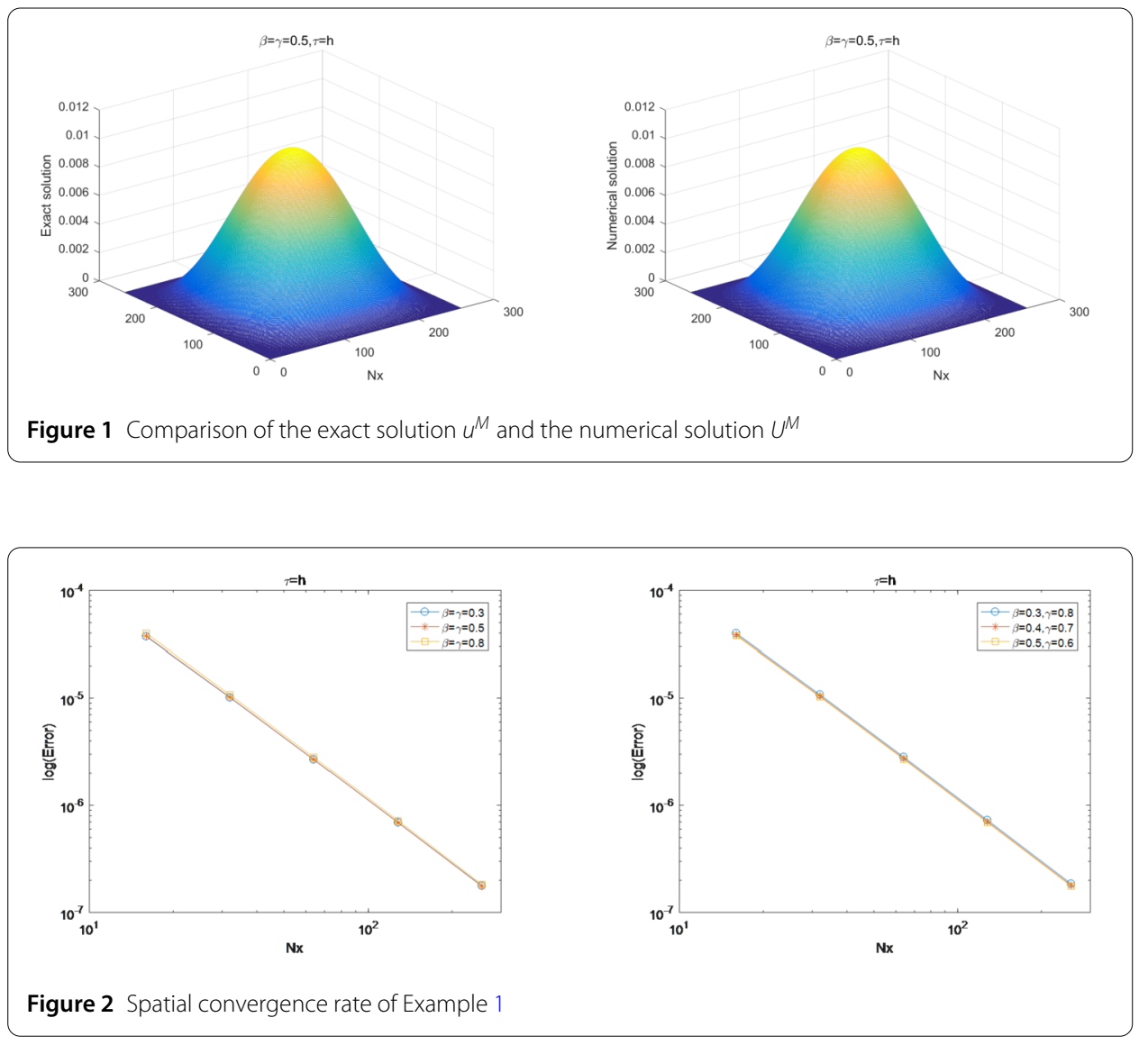

where $\Omega=(0,1) \times(0,1), 0<\beta, \gamma<1$, the source term $f=e^{t} x^{2}(1-x)^{2} y^{2}(1-y)^{2}+$ $\frac{e^{t}}{2 \cos \left(\frac{(1+\beta) \pi}{2}\right)}\left\{\frac{24}{\Gamma(4-\beta)}\left[x^{3-\beta}+(1-x)^{3-\beta}\right]-\frac{12}{\Gamma(3-\beta)}\left[x^{2-\beta}+(1-x)^{2-\beta}\right]+\frac{2}{\Gamma(2-\beta)}\left[x^{1-\beta}+(1-x)^{1-\beta}\right]\right\} y^{2}(1-$ $y)^{2}+\frac{e^{t}}{2 \cos \left(\frac{(1+\gamma) \pi}{2}\right)}\left\{\frac{24}{\Gamma(4-\gamma)}\left[y^{3-\gamma}+(1-y)^{3-\gamma}\right]-\frac{12}{\Gamma(3-\gamma)}\left[y^{2-\gamma}+(1-y)^{2-\gamma}\right]+\frac{2}{\Gamma(2-\gamma)}\left[y^{1-\gamma}+(1-\right.\right.$ $\left.\left.y)^{1-\gamma}\right]\right\} x^{2}(1-x)^{2}+e^{t}\left(4 x^{3}-6 x^{2}+2 x\right) y^{2}(1-y)^{2}+e^{t}\left(4 y^{3}-6 y^{2}+2 y\right) x^{2}(1-x)^{2}$, and the exact solution $u=e^{t} x^{2}(1-x)^{2} y^{2}(1-y)^{2}$.

The $L^{2}$-norm and convergence order of Example 2 calculated by MATLAB program are shown in Tables 7-12. As in Example 1, our final results are consistent with the theoretical analysis.

Similarly, we present two sets of images to more graphically depict the data. Figure 3 shows the errors between the numerical and exacts solutions for different $\beta, \gamma$. We can

Table $7 L^{2}$-norm and convergence rate when $\beta=\gamma=0.3,0.5,0.8$ and $\tau=h$

\begin{tabular}{|c|c|c|c|c|c|c|}
\hline \multirow[t]{2}{*}{$h$} & \multicolumn{2}{|l|}{$\beta=\gamma=0.3$} & \multicolumn{2}{|l|}{$\beta=\gamma=0.5$} & \multicolumn{2}{|l|}{$\beta=\gamma=0.8$} \\
\hline & Error & Rate & Error & Rate & Error & Rate \\
\hline $1 / 2^{4}$ & $4.4709 e-05$ & - & $4.2333 \mathrm{e}-05$ & - & $4.1909 \mathrm{e}-05$ & - \\
\hline $1 / 2^{5}$ & $1.1928 \mathrm{e}-05$ & 1.9062 & $1.1359 \mathrm{e}-05$ & 1.8979 & $1.1269 \mathrm{e}-05$ & 1.8949 \\
\hline $1 / 2^{6}$ & $3.0803 \mathrm{e}-06$ & 1.9532 & $2.9441 \mathrm{e}-06$ & 1.9479 & $2.9313 \mathrm{e}-06$ & 1.9427 \\
\hline $1 / 2^{7}$ & $7.8459 \mathrm{e}-07$ & 1.9731 & $7.5093 \mathrm{e}-07$ & 1.9711 & $7.4905 \mathrm{e}-07$ & 1.9684 \\
\hline $1 / 2^{8}$ & $1.9835 \mathrm{e}-07$ & 1.9839 & $1.8995 \mathrm{e}-07$ & 1.9831 & $1.8966 \mathrm{e}-07$ & 1.9816 \\
\hline
\end{tabular}


Table $8 L^{2}$-norm and convergence rate when $\beta=0.3,0.4,0.5, \gamma=0.8,0.7,0.6$, and $\tau=h$

\begin{tabular}{|c|c|c|c|c|c|c|}
\hline \multirow[t]{2}{*}{$h$} & \multicolumn{2}{|c|}{$\beta=0.3, \gamma=0.8$} & \multicolumn{2}{|c|}{$\beta=0.4, \gamma=0.7$} & \multicolumn{2}{|c|}{$\beta=0.5, \gamma=0.6$} \\
\hline & Error & Rate & Error & Rate & Error & Rate \\
\hline $1 / 2^{4}$ & $4.3410 \mathrm{e}-05$ & - & $4.2505 \mathrm{e}-05$ & - & $4.2049 \mathrm{e}-05$ & - \\
\hline $1 / 2^{5}$ & $1.1659 \mathrm{e}-05$ & 1.8966 & $1.1419 \mathrm{e}-05$ & 1.8962 & $1.1295 \mathrm{e}-05$ & 1.8964 \\
\hline $1 / 2^{6}$ & $3.0296 \mathrm{e}-06$ & 1.9442 & $2.9650 \mathrm{e}-06$ & 1.9453 & $2.9310 \mathrm{e}-06$ & 1.9462 \\
\hline $1 / 2^{7}$ & $7.7348 \mathrm{e}-07$ & 1.9697 & $7.5638 \mathrm{e}-07$ & 1.9708 & $7.4717 \mathrm{e}-07$ & 1.9719 \\
\hline $1 / 2^{8}$ & $1.9606 \mathrm{e}-07$ & 1.9801 & $1.9152 \mathrm{e}-07$ & 1.9816 & $1.8904 \mathrm{e}-07$ & 1.9827 \\
\hline
\end{tabular}

Table $9 L^{2}$-norm and convergence rate when $\beta=\gamma=0.3,0.5,0.6$ and $h=2^{-8}$

\begin{tabular}{|c|c|c|c|c|c|c|}
\hline \multirow[t]{2}{*}{$\tau$} & \multicolumn{2}{|l|}{$\beta=\gamma=0.3$} & \multicolumn{2}{|l|}{$\beta=\gamma=0.5$} & \multicolumn{2}{|l|}{$\beta=\gamma=0.6$} \\
\hline & Error & Rate & Error & Rate & Error & Rate \\
\hline $1 / 2^{1}$ & $1.1683 \mathrm{e}-04$ & - & $1.1465 \mathrm{e}-04$ & - & $1.1327 \mathrm{e}-04$ & - \\
\hline $1 / 2^{2}$ & $3.0989 \mathrm{e}-05$ & 1.9146 & $3.1312 \mathrm{e}-05$ & 1.8724 & $3.1468 \mathrm{e}-05$ & 1.8478 \\
\hline $1 / 2^{3}$ & $7.6545 \mathrm{e}-06$ & 2.0174 & $7.7527 \mathrm{e}-06$ & 2.0139 & $7.8036 \mathrm{e}-06$ & 2.0117 \\
\hline $1 / 2^{4}$ & $1.7903 \mathrm{e}-06$ & 2.0961 & $1.8119 \mathrm{e}-06$ & 2.0972 & $1.8193 \mathrm{e}-06$ & 2.1008 \\
\hline
\end{tabular}

Table $10 L^{2}$-norm and convergence rate when $\beta=0.3,0.4,0.5, \gamma=0.1,0.7,0.6$, and $h=2^{-8}$

\begin{tabular}{|c|c|c|c|c|c|c|}
\hline \multirow[t]{2}{*}{$\tau$} & \multicolumn{2}{|c|}{$\beta=0.3, \gamma=0.1$} & \multicolumn{2}{|c|}{$\beta=0.4, \gamma=0.7$} & \multicolumn{2}{|c|}{$\beta=0.5, \gamma=0.6$} \\
\hline & Error & Rate & Error & Rate & Error & Rate \\
\hline$\overline{1 / 2^{1}}$ & $1.1766 \mathrm{e}-04$ & - & $1.1696 \mathrm{e}-04$ & - & $1.2989 \mathrm{e}-04$ & - \\
\hline $1 / 2^{2}$ & $3.0831 \mathrm{e}-05$ & 1.9322 & $3.3372 \mathrm{e}-05$ & 1.8093 & $3.1438 \mathrm{e}-05$ & 2.0467 \\
\hline $1 / 2^{3}$ & $7.6083 e-06$ & 2.0187 & $8.1529 \mathrm{e}-06$ & 2.0333 & $7.7724 \mathrm{e}-06$ & 2.0161 \\
\hline $1 / 2^{4}$ & $1.7767 \mathrm{e}-06$ & 2.0984 & $1.8642 \mathrm{e}-06$ & 2.1288 & $1.8170 \mathrm{e}-06$ & 2.0968 \\
\hline
\end{tabular}

Table 11 Comparison of the time taken by Gauss elimination, BICGSTAB, and FAST-BICGSTAB methods when $\beta=\gamma=0.3$ and $\tau=h$

\begin{tabular}{|c|c|c|c|c|c|c|}
\hline$h$ & Method & Time 1 & Method & Time 2 & Method & Time 3 \\
\hline $1 / 2^{4}$ & Gauss & $0.013568 \mathrm{~s}$ & BICGSTAB & $0.010259 \mathrm{~s}$ & FAST-BICGSTAB & $0.262019 \mathrm{~s}$ \\
\hline $1 / 2^{5}$ & & $0.860206 \mathrm{~s}$ & & $0.349422 \mathrm{~s}$ & & $1.262514 \mathrm{~s}$ \\
\hline $1 / 2^{6}$ & & $71.711588 \mathrm{~s}$ & & $7.333496 \mathrm{~s}$ & & $6.596356 \mathrm{~s}$ \\
\hline $1 / 2^{7}$ & & $8532.462135 \mathrm{~s}$ & & $206.940085 \mathrm{~s}$ & & $28.015395 \mathrm{~s}$ \\
\hline $1 / 2^{8}$ & & - & & - & & $323.858521 \mathrm{~s}$ \\
\hline $1 / 2^{9}$ & & - & & - & & $3048.820601 \mathrm{~s}$ \\
\hline
\end{tabular}

Table 12 Comparison of the time taken by Gauss elimination, BICGSTAB, and FAST-BICGSTAB methods when $\beta=\gamma=0.8$ and $\tau=h$

\begin{tabular}{|c|c|c|c|c|c|c|}
\hline$h$ & Method & Time 1 & Method & Time 2 & Method & Time 3 \\
\hline $1 / 2^{4}$ & Gauss & $0.013328 \mathrm{~s}$ & BICGSTAB & $0.015876 \mathrm{~s}$ & FAST-BICGSTAB & $0.270941 \mathrm{~s}$ \\
\hline $1 / 2^{5}$ & & $0.857786 \mathrm{~s}$ & & $0.596994 \mathrm{~s}$ & & $1.933745 \mathrm{~s}$ \\
\hline $1 / 2^{6}$ & & $67.991373 \mathrm{~s}$ & & $25.279196 \mathrm{~s}$ & & $17.303392 \mathrm{~s}$ \\
\hline $1 / 2^{7}$ & & $7827.717809 \mathrm{~s}$ & & $1158.434168 \mathrm{~s}$ & & $123.636986 \mathrm{~s}$ \\
\hline $1 / 2^{8}$ & & - & & - & & $1683.692712 \mathrm{~s}$ \\
\hline $1 / 2^{9}$ & & - & & - & & $18272.333663 \mathrm{~s}$ \\
\hline
\end{tabular}

see that when $\tau=h=2^{-8}$, the error between the exact and numerical solutions reaches $1 \mathrm{e}-07$. Figure 4 shows the spatial convergence orders under different $\beta, \gamma$, and the data agree with the theoretical results. 

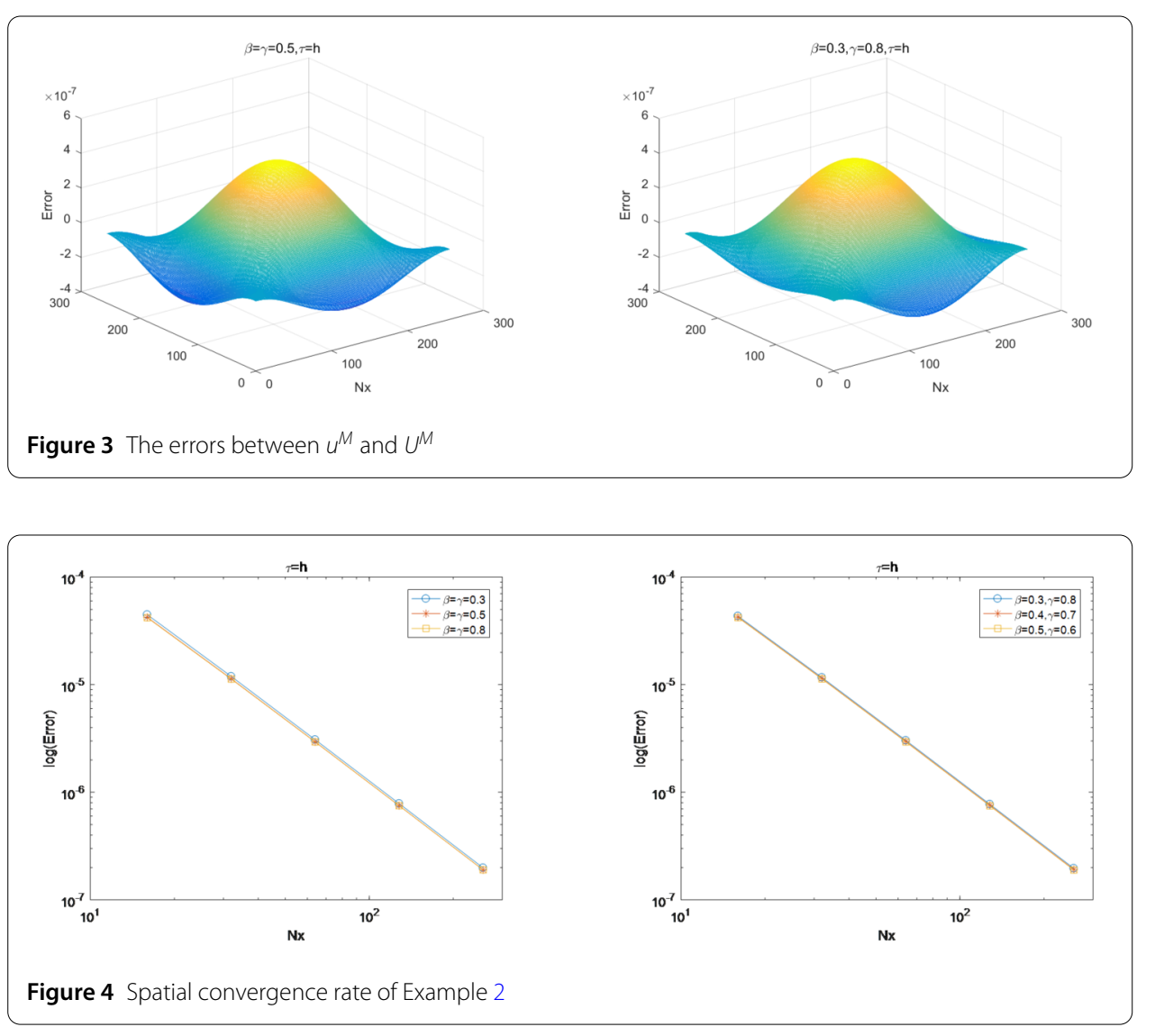

Example 3 Consider the following two-dimensional Riesz fractional convection-diffusion equation:

$$
\begin{cases}\frac{\partial u}{\partial t}-3 \frac{\partial^{1+\beta} u}{\partial|x|^{1+\beta}}-3 \frac{\partial^{1+\gamma} u}{\partial|y|^{1+\gamma}}-5 \frac{\partial u}{\partial x}-5 \frac{\partial u}{\partial y}=f, & (x, y) \in \Omega, 0<t \leq 1 \\ u=0, & (x, y) \in \partial \Omega, 0<t \leq 1 \\ u=0, & (x, y) \in \Omega, t=0\end{cases}
$$

where $\Omega=(0,1) \times(0,1), 0<\beta, \gamma<1$, the source term $f=3 t^{2} x^{2}(1-x)^{2} y^{2}(1-y)^{2}+$ $\frac{3 t^{3}}{2 \cos \left(\frac{(1+\beta) \pi}{2}\right)}\left\{\frac{24}{\Gamma(4-\beta)}\left[x^{3-\beta}+(1-x)^{3-\beta}\right]-\frac{12}{\Gamma(3-\beta)}\left[x^{2-\beta}+(1-x)^{2-\beta}\right]+\frac{2}{\Gamma(2-\beta)}\left[x^{1-\beta}+(1-x)^{1-\beta}\right]\right\} y^{2}(1-$ $y)^{2}+\frac{3 t^{3}}{2 \cos \left(\frac{(1+\gamma) \pi}{2}\right)}\left\{\frac{24}{\Gamma(4-\gamma)}\left[y^{3-\gamma}+(1-y)^{3-\gamma}\right]-\frac{12}{\Gamma(3-\gamma)}\left[y^{2-\gamma}+(1-y)^{2-\gamma}\right]+\frac{2}{\Gamma(2-\gamma)}\left[y^{1-\gamma}+(1-\right.\right.$ $\left.\left.y)^{1-\gamma}\right]\right\} x^{2}(1-x)^{2}-5 t^{3}\left(4 x^{3}-6 x^{2}+2 x\right) y^{2}(1-y)^{2}-5 t^{3}\left(4 y^{3}-6 y^{2}+2 y\right) x^{2}(1-x)^{2}$, and the exact solution $u=t^{3} x^{2}(1-x)^{2} y^{2}(1-y)^{2}$.

As in the previous two examples, we use the MATLAB program to produce the following data results (see Tables 13-14 and Fig. 5). We find that the calculated data are consistent with the theoretical results obtained from our previous analysis.

\section{Conclusion}

In this paper, we have successfully given the Crank-Nicolson finite volume element scheme for two-dimensional Riesz space-fractional convection-diffusion equations. We use the finite volume element scheme to discretize the space-fractional derivatives and the 
Table $13 L^{2}$-norm and convergence rate when $\beta=\gamma=0.3,0.5,0.8$ and $\tau=h$

\begin{tabular}{|c|c|c|c|c|c|c|}
\hline \multirow[t]{2}{*}{$h$} & \multicolumn{2}{|l|}{$\beta=\gamma=0.3$} & \multicolumn{2}{|l|}{$\beta=\gamma=0.5$} & \multicolumn{2}{|l|}{$\beta=\gamma=0.8$} \\
\hline & Error & Rate & Error & Rate & Error & Rate \\
\hline $1 / 2^{4}$ & $1.5804 \mathrm{e}-05$ & - & $1.4156 \mathrm{e}-05$ & - & $1.2805 \mathrm{e}-05$ & - \\
\hline $1 / 2^{5}$ & $4.2150 \mathrm{e}-06$ & 1.9067 & $3.8236 \mathrm{e}-06$ & 1.8884 & $3.5015 \mathrm{e}-06$ & 1.8707 \\
\hline $1 / 2^{6}$ & $1.0833 \mathrm{e}-06$ & 1.9601 & $9.9123 \mathrm{e}-07$ & 1.9476 & $9.1644 \mathrm{e}-07$ & 1.9339 \\
\hline $1 / 2^{7}$ & $2.7406 \mathrm{e}-07$ & 1.9829 & $2.5219 \mathrm{e}-07$ & 1.9747 & $2.3463 \mathrm{e}-07$ & 1.9657 \\
\hline $1 / 2^{8}$ & $6.8920 \mathrm{e}-08$ & 1.9915 & $6.3652 \mathrm{e}-08$ & 1.9862 & $5.9574 \mathrm{e}-08$ & 1.9776 \\
\hline
\end{tabular}

Table $14 L^{2}$-norm and convergence rate when $\beta=0.3,0.4,0.5, \gamma=0.1,0.5,0.6$, and $h=2^{-8}$

\begin{tabular}{|c|c|c|c|c|c|c|}
\hline \multirow[t]{2}{*}{$\bar{\tau}$} & \multicolumn{2}{|c|}{$\beta=0.3, \gamma=0.1$} & \multicolumn{2}{|c|}{$\beta=0.4, \gamma=0.5$} & \multicolumn{2}{|c|}{$\beta=0.5, \gamma=0.6$} \\
\hline & Error & Rate & Error & Rate & Error & Rate \\
\hline $1 / 2^{1}$ & $2.9489 \mathrm{e}-04$ & - & $2.9544 \mathrm{e}-04$ & - & $2.9512 \mathrm{e}-04$ & - \\
\hline $1 / 2^{2}$ & $7.2841 \mathrm{e}-05$ & 2.0174 & $7.3103 e-05$ & 2.0149 & $7.3263 \mathrm{e}-05$ & 2.0101 \\
\hline $1 / 2^{3}$ & $1.8106 \mathrm{e}-05$ & 2.0083 & $1.8163 \mathrm{e}-05$ & 2.0089 & $1.9625 \mathrm{e}-05$ & 1.9004 \\
\hline $1 / 2^{4}$ & $4.4716 \mathrm{e}-06$ & 2.0176 & $4.4856 \mathrm{e}-06$ & 2.0176 & $4.5131 \mathrm{e}-06$ & 2.1205 \\
\hline
\end{tabular}

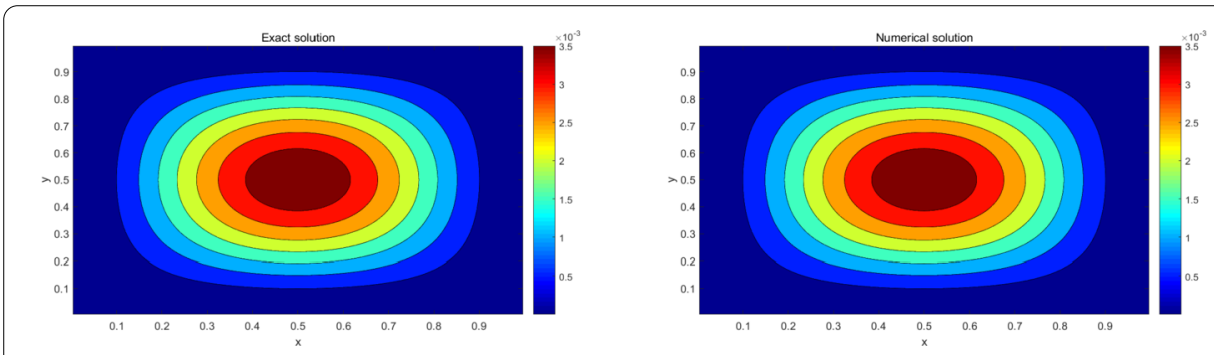

Figure 5 Comparison of color contour plots of the exact solution $u^{M}$ and the numerical solution $U^{M}$ when $\beta=\gamma=0.3$ and $\tau=h=2^{-8}$

$\mathrm{CN}$-scheme to approximate the time derivatives. We show that the fully discrete scheme is stable and convergent. Finally, we verify the correctness and validity of the theoretical analysis through three examples.

\section{Acknowledgements}

The authors would like to express their sincere thanks to the reviewers for their valuable comments and suggestions, which have contributed to the improvement of the original manuscript.

\section{Funding}

This work is supported by the Natural Science Foundation of Shandong Province of China (No. ZR2017MA020).

\section{Availability of data and materials}

Not applicable.

\section{Competing interests}

The authors declare that they have no competing interests.

\section{Authors' contributions}

Both authors read and approved the final manuscript.

\section{Publisher's Note}

Springer Nature remains neutral with regard to jurisdictional claims in published maps and institutional affiliations.

Received: 19 April 2021 Accepted: 18 July 2021 Published online: 16 August 2021

\section{References}

1. Hilfer, R.: Applications of Fractional Calculus in Physics. World Scientific, Singapore (2000) 
2. Lotfy, K.: A novel solution of fractional order heat equation for photothermal waves in a semiconductor medium with a spherical cavity. Chaos Solitons Fractals 99, 233-242 (2017)

3. Nikan, O., Jafari, H., Golbabai, A.: Numerical analysis of the fractional evolution model for heat flow in materials with memory. Alex. Eng. J. 59(4), 2627-2637 (2020)

4. Ganji, R.M., Jafari, H., Baleanu, D.: A new approach for solving multi variable orders differential equations with Mittag-Leffler kernel. Chaos Solitons Fractals 130, 109405 (2020)

5. Tuan, N.H., Aghdam, Y.E., Jafari, H., Mesgarani, H.: A novel numerical manner for two-dimensional space fractional diffusion equation arising in transport phenomena. Numer. Methods Partial Differ. Equ. 37, 1397-1406 (2021)

6. Metzler, R., Klafter, J.: The random walk's guide to anomalous diffusion: a fractional dynamics approach. Phys. Rep. $339(1), 1-77(2000)$

7. Gorenflo, R., Mainardi, F.: Approximation of Levy-Feller diffusion by random walk. Z. Anal. Anwend. 18(2), 231-246 (1999)

8. Feller, W.: An Introduction to Probability Theory and Its Application, 2nd edn. Wiley, New York (1971)

9. Hejazi, H., Moroney, T., Liu, F.: Stability and convergence of a finite volume method for the space fractional advection-dispersion equation. J. Comput. Appl. Math. 255, 684-697 (2014)

10. Yang, Q., Turner, I., Moroney, T., Liu, F.: A finite volume scheme with preconditioned Lanczos method for two-dimensional space-fractional reaction-diffusion equations. Appl. Math. Model. 38(15-16), 3755-3762 (2014)

11. Jia, J., Wang, H.: A fast finite volume method for conservative space-fractional diffusion equations in convex domains. J. Comput. Phys. 310, 63-84 (2016)

12. Zhang, T., Guo, Q.: The finite difference/finite volume method for solving the fractional diffusion equation. J. Comput. Phys. 375, 120-134 (2018)

13. Liu, H., Cheng, A., Wang, H.: A parareal finite volume method for variable-order time-fractional diffusion equations. J. Sci. Comput. 85(1), 19 (2020)

14. Liu, F., Zhuang, P., Turner, I., Burrage, K., Anh, V.: A new fractional finite volume method for solving the fractional diffusion equation. Appl. Math. Model. 38(15-16), 3871-3878 (2014)

15. Feng, L.B., Zhuang, P., Liu, F., Turner, I.: Stability and convergence of a new finite volume method for a two-sided space-fractional diffusion equation. Appl. Math. Comput. 257, 52-65 (2015)

16. Karaa, S., Mustapha, K., Pani, A.K.: Finite volume element method for two-dimensional fractional subdiffusion problems. IMA J. Numer. Anal. 37(2), 945-964 (2017)

17. Fu, H., Liu, H., Wang, H.: A finite volume method for two-dimensional Riemann-Liouville space-fractional diffusion equation and its efficient implementation. J. Comput. Phys. 388, 316-334 (2019)

18. Fu, H., Sun, Y., Wang, H., Zheng, X.: Stability and convergence of a Crank-Nicolson finite volume method for space fractional diffusion equations. Appl. Numer. Math. 139, 38-51 (2019)

19. Zhao, J., Fang, Z., Li, H., Liu, Y.: A Crank-Nicolson finite volume element method for time fractional Sobolev equations on triangular grids. Mathematics 8(9), 1591 (2020)

20. Zhao, J., Fang, Z., Li, H., Liu, Y.: Finite volume element method with the WSGD formula for nonlinear fractional mobile/immobile transport equations. Adv. Differ. Equ. 2020, 360 (2020)

21. Wang, H., Wang, K., Sircar, T.: A direct $O(N \log 2 N)$ finite difference method for fractional diffusion equations. J. Comput. Phys. 229(21), 8095-8104 (2010)

22. Sousa, E., Li, C.: A weighted finite difference method for the fractional diffusion equation based on the Riemann-Liouville derivative. Appl. Numer. Math. 90, 22-37 (2015)

23. Arshad, S., Baleanu, D., Huang, J., Al Qurashi, M., Tang, Y., Zhao, Y.: Finite difference method for time-space fractional advection-diffusion equations with Riesz derivative. Entropy 20(5), 321 (2018)

24. Mirzaee, F. Samadyar, N.: Combination of finite difference method and meshless method based on radial basis functions to solve fractional stochastic advection-diffusion equations. Eng. Comput. 36(4), 1673-1686 (2020)

25. Anley, E.F., Zheng, Z.: Finite difference approximation method for a space fractional convection-diffusion equation with variable coefficients. Symmetry 12(3), 485 (2020)

26. She, Z., Qu, H., Liu, X.: Stability and convergence of finite difference method for two-sided space-fractional diffusion equations. Comput. Math. Appl. 89, 78-86 (2021)

27. Lian, Y., Ying, Y., Tang, S., Lin, S., Wagner, G.J., Liu, W.K.: A Petrov-Galerkin finite element method for the fractional advection-diffusion equation. Comput. Methods Appl. Mech. Eng. 309, 388-410 (2016)

28. Feng, L.B., Zhuang, P., Liu, F., Turner, I., Gu, Y.T.: Finite element method for space-time fractional diffusion equation. Numer. Algorithms 72(3), 749-767 (2016)

29. Çelik, C., Duman, M.: Finite element method for a symmetric tempered fractional diffusion equation. Appl. Numer. Math. 120, 270-286 (2017)

30. Abbaszadeh, M., Dehghan, M.: Analysis of mixed finite element method (MFEM) for solving the generalized fractional reaction-diffusion equation on nonrectangular domains. Comput. Math. Appl. 78(5), 1531-1547 (2019)

31. Gao, J., Zhao, M., Du, N., Guo, X., Wang, H., Zhang, J.: A finite element method for space-time directional fractional diffusion partial differential equations in the plane and its error analysis. J. Comput. Appl. Math. 362, 354-365 (2019)

32. Bueno-Orovio, A., Kay, D., Burrage, K.: Fourier spectral methods for fractional-in-space reaction-diffusion equations. BIT Numer. Math. 54(4), 937-954 (2014)

33. Pindza, E., Owolabi, K.M.: Fourier spectral method for higher order space fractional reaction-diffusion equations Commun. Nonlinear Sci. Numer. Simul. 40, 112-128 (2016)

34. Chen, H., Lü, S., Chen, W.: Spectral methods for the time fractional diffusion-wave equation in a semi-infinite channel. Comput. Math. Appl. 71(9), 1818-1830 (2016)

35. Çelik, C., Duman, M.: Crank-Nicolson method for the fractional diffusion equation with the Riesz fractional derivative. J. Comput. Phys. 231(4), 1743-1750 (2012)

36. Pindza, E., Owolabi, K.M.: Fourier spectral method for higher order space fractional reaction-diffusion equations. Commun. Nonlinear Sci. Numer. Simul. 40, 112-128 (2016)

37. Zhu, X.G., Nie, Y.F., Zhang, W.W.: An efficient differential quadrature method for fractional advection-diffusion equation. Nonlinear Dyn. 90(3), 1807-1827 (2017)

38. Dehghan, M., Abbaszadeh, M., Deng, W.: Fourth-order numerical method for the space-time tempered fractional diffusion-wave equation. Appl. Math. Lett. 73, 120-127 (2017) 
39. Shen, J., Sheng, C.: An efficient space-time method for time fractional diffusion equation. J. Sci. Comput. 81 (2), 1088-1110 (2019)

40. Feng, R., Liu, Y., Hou, Y., Li, H., Fang, Z.: Mixed element algorithm based on a second-order time approximation scheme for a two-dimensional nonlinear time fractional coupled sub-diffusion model. Eng. Comput. (2020)

41. Horn, R.A., Johnson, C.R.: Topics in Matrix Analysis. Cambridge University Press, Cambridge (1994)

42. Quarteroni, A., Sacco, R., Saleri, F.: Numerical Mathematics, vol. 37, 2nd edn. Springer, Berlin (2007)

43. Jin, X.: Preconditioning Techniques for Toeplitz Systems. Higher Education Press, Beijing (2010)

Submit your manuscript to a SpringerOpen ${ }^{\circ}$ journal and benefit from:

- Convenient online submission

- Rigorous peer review

- Open access: articles freely available online

- High visibility within the field

- Retaining the copyright to your article

Submit your next manuscript at $\gg$ springeropen.com 